JOURNAL OF THE

AMERICAN MATHEMATICAL SOCIETY

Volume 12, Number 4, Pages 1179-1212

S 0894-0347(99)00312-4

Article electronically published on July 6, 1999

\title{
COMPACT SUBSETS OF THE FIRST BAIRE CLASS
}

\author{
STEVO TODORČEVIĆ
}

Perhaps the earliest results about pointwise compact sets of Baire class-1 functions are the two selection theorems of E. Helly found in most of the standard texts on real variable (see, e.g., $[\mathrm{Lo}],[\mathrm{N}]$ ). These two theorems are really theorems about a particular example of a compact set of Baire class-1 functions known today as Helly space, the space of all nondecreasing functions from the unit interval $I=[0,1]$ into itself. More recently, the notion of Baire class-1 function turned out to also be important in some areas of functional analysis (see [R3]). For example, Odell and Rosenthal $[\mathrm{OR}]$ showed that the double dual of a separable Banach space $E$ with the weak* topology consists only of Baire class- 1 functions defined on the unit ball of $E^{*}$ if and only if the space $E$ contains no subspace isomorphic to $\ell^{1}$. This resulted in a renewed interest in this class of spaces. For example, building on the work of Rosenthal [R2], Bourgain, Fremlin and Talagrand [BFT] proved analogues of the two theorems of Helly for the whole first Baire class. Using their results Godefroy [Go] showed that this class of spaces enjoys some interesting permanence properties. For example, if a compact space $K$ is representable as a compact set of Baire class-1 functions, then so is $P(K)$, the space of all Radon probability measures on $K$ with the weak* topology. Some further permanence properties of this class of spaces were obtained by Marciszewski ([M1], [M2]) and an excellent survey of the early results is given by $\mathrm{R}$. Pol [Po2]. Our paper is an attempt towards a fine structure theory of compact subsets of first Baire class. The first result that we give is a positive answer to a natural question one usually asks in such a context.

Theorem 1. Every compact subset of first Baire class contains a dense metrizable subspace.

An interesting feature of this result and its proof is that it necessarily requires methods quite different from the classical ones which go through Namioka's analysis of joint versus separate continuity (see $[\mathrm{Na}]$ ) and which therefore usually produce dense completely metrizable subspaces. Existence of a dense metrizable subspace of $K$ means that $K$ contains many $G_{\delta}$ points, a fact first established by Bourgain [Bo]. Note that from the fact that every compact set $K$ of first Baire class contains a $G_{\delta}$ point one can conclude that every such $K$ contains a set of $G_{\delta}$ points which is everywhere of second category in $K$. Such a generous conclusion cannot be given for dense metrizable subspaces, revealing the striking difference between the two results. For example, there exists a $K$ of the first Baire class such that every metrizable subspace of $K$ is countable. One example of such $K$ is the split interval $S(I)$, the lexicographically ordered product $I \times 2$ of the unit interval $I=[0,1]$ and the

Received by the editors January 20, 1997 and, in revised form, April 20, 1999.

1991 Mathematics Subject Classification. Primary 26A21, 28A05, 28A20, 05D10, 03E05, 03E15, 54H05, 46B25, 46B45. 
two-element ordering $2=\{0,1\}$ which can naturally be represented as a subspace of the Helly space described above. An immediate consequence of Theorem 1 is the fact that every Radon probability measure on some compact set $K$ of the first Baire class has a separable support. This fact was first proved by Godefroy [Go] using some deep results from [BFT]. The methods used in the proof of Theorem 1 are likely to lead to a definite result in this direction which will be examined elsewhere. Here we just mention that even at this stage they are good enough to supply a proof of the following result of J. Bourgain given without a proof in [Ta, 14-2-2].

Theorem 2. If $K$ is a compact subset of first Baire class, then $L^{1}(K, \mu)$ is separable for every Radon probability measure $\mu$ on $K$.

Note that the projection from the split interval $S(I)$ to the unit interval $I$ is a two-to-one continuous mapping. Rather unexpectedly, the following result shows that this property of the split interval $S(I)$ is shared by all compact sets of the first Baire class provided they are not very wild.

Theorem 3. Every compact subset of the first Baire class which is not metric itself contains either an uncountable discrete subspace or is an at most two-to-one continuous preimage of a compact metric space.

Our next result shows an even more striking role of the split interval in this class of spaces.

Theorem 4. Every compact subset of the first Baire class which is not metric itself contains either an uncountable discrete subspace or a homeomorphic copy of the split interval.

Theorems 3 and 4 will be consequences of an even finer analysis of compact sets of the first Baire class which we are now going to discuss. Even at the early stage of the development of the theory it was clear that separability is the property that naturally splits this class into two. Indeed many compacta of the first Baire class are separable. For example, the Helly space, the split interval, or the double dual ball of a separable Banach space which contains no isomorphic copy of $\ell^{1}$ are all separable. While nonseparable compacta of the first Baire class can be quite pathological, the separable ones are all "definable". In [Bo], J. Bourgain gives an example of a compact set of first Baire class not contained in any separable one. (Pathologies of this kind give us some explanation to why some care is needed in the preservation result of $\S \mathrm{A}$ below.) An indication that separable compacta of the first Baire class are well behaved is given in a characterization of this class of spaces due to Godefroy [Go]. It states that a separable compactum $K$ is representable as a compact set of the first Baire class if and only if every subspace $E$ of $\ell^{\infty}$ isomorphic to the Banach space $C(K)$ is analytic as a subspace of the Tychonoff cube $\mathbb{R}^{\mathbb{N}}$. Building on an earlier work of C. Stegall [St], this enabled Godefroy together with Louveau and Talagrand (see [GL], [GT]) to lift some of the classical theory of analytic sets to the context of the Banach space $C(K)$, or more generally, the class of "representable Banach spaces". Our strategy here will be to enrich the classical theory of analytic sets by a new combinatorial feature (first exposed in [To1]), and use this to analyze the topological structure of $K$ rather than $C(K)$. In fact, the reader familiar with our analysis of partition problems in topology (see [To1], $[\mathrm{Gr}]$ ) will not have difficulties in realizing where the hints to our dichotomies and trichotomies are coming from. 
To state the finer analysis we need to recall a few more examples of compact subsets of the first Baire class. For a given point $x$ in some Polish space $X$, let $\delta_{x}$ be its Dirac function $\delta_{x}: X \rightarrow 2$, i.e., $\delta_{x}(y)=0$ iff $x \neq y$. Clearly, each $\delta_{x}$ is a Baire class- 1 function on $X$ and

$$
A(X)=\left\{\delta_{x}: x \in X\right\} \cup\{\overline{0}\}
$$

is a compact set of the first Baire class homeomorphic to the one-point compactification of the discrete space $\left\{\delta_{x}: x \in X\right\}$. This is clearly a nonseparable example but it can be supplemented to one (see [M1], [Po2]). This is most easily seen in the case when $X=2^{\mathbb{N}}$. For if for a finite sequence $s \in 2^{<\mathbb{N}}$ we let $\delta_{s}$ be the characteristic function of the set of all $x \in 2^{<\mathbb{N}}$ which end-extend $s$, we get that $\left\{\delta_{s}: s \in 2^{<\mathbb{N}}\right\}$ is a countable dense subset of the compact space

$$
A\left(2^{\mathbb{N}}\right) \cup\left\{\delta_{s}: s \in 2^{<\mathbb{N}}\right\}
$$

of the first Baire class on $2^{\mathbb{N}}$. In fact, $\left\{\delta_{s}: s \in 2^{<\mathbb{N}}\right\}$ is discrete so $A\left(2^{\mathbb{N}}\right)$ appears there as the remainder of its compactification. Note that $A\left(2^{\mathbb{N}}\right)$ is very far from being an at most two-to-one preimage of a metric compactum. Since Theorem 3 shows that in our context this is a very natural class of spaces, we introduce a special name for it. Thus, we say that $K$ is a premetric compactum of degree at most 2 if $K$ is an at most two-to-one continuous preimage of a metric compactum. The most famous and best behaved such premetric compactum (which is not metric itself) is of course the split interval. But there is another natural example of a premetric compactum of degree 2. This is the Alexandroff duplicate $D(M)$ of some metric compactum $M$. It is the space on the product $M \times 2$ where $M \times\{0\}$ is homeomorphic to $M$, the points of $M \times\{1\}$ are all isolated, while a typical open neighborhood of some $(x, 0)$ has the form

$$
(U \times 2) \backslash F
$$

for some open neighborhood $U$ of $x$ in $M$ and some finite set $F$ of points from $M \times\{1\}$. To see that $D(M)$ is representable as a compact subset of the first Baire class, consider the mapping $\Phi: M \times 2 \rightarrow M \times A(M)$ defined by

$$
\Phi(x, 0)=(x, \overline{0}) \text { and } \Phi(x, 1)=\left(x, \delta_{x}\right) .
$$

It is easily checked that $\Phi$ is a topological embedding. Note that $D(M)$ is nonseparable (if $M$ is uncountable) but it can be supplemented to a separable compactum of the first Baire class by adding to it only countably many (isolated) points. This is again most easily seen in the case when $M=2^{\mathbb{N}}$. Keeping in mind the above representation of $D\left(2^{\mathbb{N}}\right)$ as a subspace of the product $2^{\mathbb{N}} \times A\left(2^{\mathbb{N}}\right)$ as well as the way the compact set $A\left(2^{\mathbb{N}}\right)$ was supplemented to a separable space, consider

$$
\Phi\left[D\left(2^{\mathbb{N}}\right)\right] \cup\left\{\left(e, \delta_{e \uparrow n}\right): e \in E, n \in \mathbb{N}\right\}
$$

where $E$ is some fixed countable dense subset of the Cantor set $2^{\mathbb{N}}$. We are now ready to state our results about separable compact sets of the first Baire class.

Theorem 5. Every separable compact subset of the first Baire class satisfies one of the following two alternatives:

(a) $K$ contains a discrete subspace of size continuum.

(b) $K$ is a premetric compactum of degree at most 2 . 
The class of compacta satisfying the alternative (a) is at the moment hard to grasp. We expect that some information about this class will come from the development of the local theory. On the other hand, the class of premetric compacta of degree 2 is, rather unexpectedly, largely determined by two of its most typical members:

Theorem 6. Every separable premetric compactum $K$ of degree at most 2 and of the first Baire class satisfies one of the following alternatives:

(a) $K$ itself is a metric compactum.

(b) $K$ contains the duplicate of the Cantor set.

(c) $K$ contains the split interval.

Note that removing some of the isolated points from $D\left(2^{\mathbb{N}}\right)$ still makes it a compact set of the first Baire class. Of course by removing too many such points one may obtain a (nonmetrizable) compactum which contains no copy of $D\left(2^{\mathbb{N}}\right)$. This shows that in Theorem 6 the separability is an essential assumption. (This is of course even more clear in the case of Theorem 5.) Note also that the analogous manipulation with the split interval may lead to a nonmetric compactum which contains no copy of the split interval itself, and so by Theorem 6 it is not representable as a compact set of the first Baire class. There are striking differences between the two preimages $S(I)$ and $D(I)$ of the unit interval $I$. While the split interval shares many of the pleasant properties of the unit interval itself such as for example hereditary separability, the Alexandroff duplicate does not have any of these nice properties. The following further refinement of Theorem 6 shows that the duplicate is a critical space with such a wild topological behavior.

Theorem 7. The following are equivalent for every separable premetric compactum of degree at most 2 and of the first Baire class:

(a) Every subspace of $K$ is separable.

(b) Every closed subset of $K$ is $G_{\delta}$ in $K$.

(c) $K$ contains no duplicate of the Cantor set.

We note that the equivalence between (a) and (b) of this theorem was first established by R. Pol [Po2, Theorem 3.3].

Theorems 6 and 7 show that the split interval and the duplicate of the Cantor set are "critical" spaces that characterize certain classes of compacta of the first Baire class. The one-point compactification of the discrete space of size continuum is another critical space that shows up when trying to develop a local theory of compact subsets of the first Baire class. We have seen above that this space has a natural copy, $A(X)$, inside the first Baire class over any uncountable Polish space $X$ with the point at infinity represented by the zero function $\overrightarrow{0}$ while the other points are the Dirac functions $\delta_{x}$. The point at infinity is the only non- $G_{\delta}$-point and this is witnessed in a rather extreme fashion. From the examples we have seen so far one may conclude that compact subsets of the first Baire class are frequently first countable or at least they have many $G_{\delta}$-points. That this is not an accident follows from the following result of J. Bourgain [Bo].

Theorem 8. Every compact subset of the first Baire class contains a dense set of $G_{\delta}$-points.

The following result shows that the one-point compactification $A\left(2^{\mathbb{N}}\right)$ is a critical space that characterizes non- $G_{\delta}$-points in separable compacta of the first Baire class. 
Theorem 9. Suppose $K$ is a separable compact subset of the first Baire class and that $z$ is a non- $G_{\delta}$-point of $K$. Then $K$ contains a copy of $A\left(2^{\mathbb{N}}\right)$ with $z$ as its point at infinity.

This result solves a problem of R. Pol [Po2] about characters of points in separable compact subsets of the first Baire class. It shows that there exist only three possible characters. Partial results to the effect that the character of a non- $G_{\delta}$-point must be large have been given by Pol [Po2] and Krawczyk [Kr].

As indicated before, the proofs of these nine results involve a number of quite diverse methods and ideas which we find as interesting as the results themselves. The combinatorial approach of Rosenthal ([R1], [R2], [R3]) is supplemented by the new parameterized Ramsey theory, while the really new idea is the use of forcing which is not only crucially employed in some of the proofs (especially of Theorem 1), but was also the guiding force behind the discovery of these results.

\section{A. Preserving Relative Compactness}

For a given Polish space $X$ let $B_{1}(X)$ denote the family of all Baire class-1 real-valued functions defined on $X$. We take $B_{1}(X)$ always with the topology of pointwise convergence, i.e., the topology induced from the Tychonoff cube $\mathbb{R}^{X}$. A pointwise bounded set $F \subseteq B_{1}(X)$ is relatively compact in $B_{1}(X)$ if its closure in $\mathbb{R}^{X}$ is actually a subset of $B_{1}(X)$, i.e., a compact set of the first Baire class. In this section we are interested in the effect of forcing on the notion of relative compactness. So let $\mathcal{B}$ be a given forcing notion, i.e., a complete boolean algebra, fixed from now on. We first need to make some sense out of $X$ as well as $B_{1}(X)$ when we pass to the forcing extension $V^{(\mathcal{B})}$ and the reader is referred to the excellent survey article by $\mathrm{D}$. Scott $[\mathrm{S}]$ which contains all the technicalities about forcing needed here. In fact, it will be convenient to replace $X$ by the Baire space $\mathbb{N}^{\mathbb{N}}$, a surjectively universal Polish space. This can be done because $B_{1}(X)$ can be topologically embedded into $B_{1}\left(\mathbb{N}^{\mathbb{N}}\right)$ for every Polish space $X$, so there will be no loss of generality. The forcing extension $V^{(\mathcal{B})}$ has its own versions $\left(\mathbb{N}^{\mathbb{N}}\right)_{\mathcal{B}}$ and $\mathbb{R}_{\mathcal{B}}$ of $\mathbb{N}^{\mathbb{N}}$ and $\mathbb{R}$, respectively, which in general are bigger. So we will need to extend every member $f$ of $B_{1}\left(\mathbb{N}^{\mathbb{N}}\right)$ to a function $\hat{f}:\left(\mathbb{N}^{\mathbb{N}}\right)_{\mathcal{B}} \rightarrow \mathbb{R}_{\mathcal{B}}$, hopefully still of the first Baire class. This is done in a quite natural and standard way (see $[\mathrm{S}]$ ). We first extend a continuous function $f: \mathbb{N}^{\mathbb{N}} \rightarrow \mathbb{R}$ by identifying it with the monotone sequence

$$
O_{q}(f)=\left\{x \in \mathbb{N}^{\mathbb{N}}: f(x)<q\right\} \quad(q \in \mathbb{Q})
$$

of open subsets of the Baire space. An open subset $O$ of $\mathbb{N}^{\mathbb{N}}$ is usually coded by the family

$$
(O)=\left\{s \in \mathbb{N}^{<\mathbb{N}}:[s] \subseteq O\right\}
$$

of certain finite sequences of integers, where for a given sequence $s \in \mathbb{N}<\mathbb{N}$ we let

$$
[s]=\left\{x \in \mathbb{N}^{\mathbb{N}}: x \text { end-extends } s\right\} .
$$

Decoding each $\left(O_{q}(f)\right)(q \in \mathbb{Q})$ in the forcing extension $V^{(\mathcal{B})}$, we get a monotone sequence

$$
\widehat{O_{q}(f)}=\bigcup_{s \in\left(O_{q}(f)\right)}[s] \quad(q \in \mathbb{Q})
$$


of open sets of the Baire space $\left(\mathbb{N}^{\mathbb{N}}\right)_{\mathcal{B}}$. The sequence $\widehat{O_{q}(f)}(q \in \mathbb{Q})$ gives us a map $\hat{f}:\left(\mathbb{N}^{\mathbb{N}}\right)_{\mathcal{B}} \rightarrow \mathbb{R}_{\mathcal{B}}$ as follows:

$$
\hat{f}(x)=\inf \left\{q \in \mathbb{Q}: x \in \widehat{O_{q}(f)}\right\} .
$$

It is easily seen that $\hat{f}$ is continuous and that its restriction to the old elements of $\mathbb{N}^{\mathbb{N}}$ agrees with $f$. To extend a Baire class- 1 function $f$ choose a sequence $\left\{f_{n}\right\}$ of continuous functions converging pointwise to $f$. It is not hard to see that the sequence $\left\{\hat{f}_{n}\right\}$ of extensions is still pointwise convergent, so we define $\hat{f}$ to be the pointwise limit of $\{\hat{f}\}$. It is also not hard to see that for any other sequence $\left\{g_{n}\right\}$ of continuous functions converging to $f$ the sequences of extensions $\left\{\hat{f}_{n}\right\}$ and $\left\{\hat{g}_{n}\right\}$ converge to the same limit, so there is no ambiguity in this definition. The reader who is encountering this for the first time is strongly urged to recheck these two facts since from now on we are going to make many similar moves. We are now ready to state and prove the main result of this section.

Lemma 1. Suppose $F$ is a pointwise bounded relatively compact subset of $B_{1}\left(\mathbb{N}^{\mathbb{N}}\right)$. Then every member of $\mathcal{B}^{+}$forces that the family $\hat{F}=\{\hat{f}: f \in F\}$ of extensions is also relatively compact.

Proof. Suppose that $\hat{F}$ is not relatively compact in $V^{(\mathcal{B})}$ and pick an accumulation point $\dot{g}$ of $\hat{F}$ which is not of Baire class-1. By the Baire characterization theorem (see $[\mathrm{Ba}]$ ) there exist a perfect set $P$, two countable dense subsets $\left\{\dot{x}_{n}\right\}_{n=1}^{\infty}$ and $\left\{\dot{y}_{n}\right\}_{n=1}^{\infty}$ of $P$, and two rationals $\delta<\varepsilon$ such that for all $n$,

$$
\dot{g}\left(\dot{x}_{n}\right)<\delta \text { and } \dot{g}\left(\dot{y}_{n}\right)>\varepsilon \text {. }
$$

Having fixed the $\mathcal{B}$-names for these objects, let $a$ be a condition of $\mathcal{B}^{+}$which forces these facts about them and which decides the two rationals $\varepsilon$ and $\delta$. Extend $a$ to a condition $b$ such that for some $f_{0} \in \hat{F}, b$ forces that

$$
\hat{f}_{0}\left(\dot{x}_{0}\right)<\delta \text { and } \hat{f}_{0}\left(\dot{y}_{0}\right)>\varepsilon \text {. }
$$

Such $b$ and $f_{0}$ can be found because $a$ forces that $\dot{g}$ is an accumulation point of $\hat{F}$ and because $a$ forces (A1). Pick a sequence $\left\{f_{0}^{k}\right\}$ of continuous functions converging to $f_{0}$. Extending $b$ further we can find $k_{0}$ such that $b$ forces

$$
\hat{f}_{0}^{k}\left(\dot{x}_{0}\right)<\delta \text { and } \hat{f}_{0}^{k}\left(\dot{y}_{0}\right)>\varepsilon \text { for all } k \geq k_{0} .
$$

Extend $b$ still further and find an integer $i_{0}$, a finite set $T(0) \subseteq \mathbb{N}^{i_{0}}$, and for every $t \in T(0)$ two integers $m_{t}$ and $n_{t}$ such that $b$ forces

(A4) $T(0)=\left\{x\left\lceil i_{0}: x \in P\right\}\right.$,

(A5) $\dot{x}_{m_{t}}\left\lceil i_{0}=t=\dot{y}_{n_{t}}\left\lceil i_{0}\right.\right.$.

Moreover, we assume that $b$ decides $\dot{x}_{0}\left\lceil i_{0}\right.$ and $\dot{y}_{0}\left\lceil i_{0}\right.$ and if $s$ and $t$ are the respective decisions, then

$$
f_{0}^{k_{0}}[s] \subseteq(-\infty, \delta) \text { and } f_{0}^{k_{0}}[t] \subseteq(\varepsilon,+\infty) .
$$

Finally we let $a_{0}$ be this $b$ and go to the second stage of the construction. Proceeding in this way we build a sequence $\left\{a_{n}\right\}$ of stronger and stronger conditions of $\mathcal{B}^{+}$, a sequence $\left\{f_{n}\right\}$ of elements of $F$ and for each $n$ a sequence $\left\{f_{n}^{k}\right\}$ of continuous functions converging to $f_{n}$, two increasing sequences of integers $\left\{k_{n}\right\},\left\{i_{n}\right\}$ and for each $n$ a finite set $T(n) \subseteq \mathbb{N}^{i_{n}}$ and sequences $m_{t}(t \in T(n))$ and $n_{t}(t \in T(n))$ of 
integers such that

(A7) every node of $T(n)$ has at least two incomparable extensions in $T(n+1)$,

(A8) $a_{n}$ forces $T(n)=\left\{x\left\lceil i_{n}: x \in \dot{P}\right\}\right.$,

(A9) $a_{n}$ forces $\dot{x}_{m_{t}}\left\lceil i_{n}=t=\dot{y}_{n_{t}}\left\lceil i_{n}\right.\right.$ for every $t \in T(n)$,

(A10) $a_{n}$ decides $\dot{x}_{0}\left\lceil i_{n}, \ldots, \dot{x}_{n} \uparrow i_{n}\right.$ to be equal to $s_{0}^{n}, \ldots, s_{n}^{n}$, respectively,

(A11) $f_{m}^{k_{n}}\left[s_{\ell}^{n}\right] \subseteq(-\infty, \delta)$ for all $\ell \leq m \leq n$,

(A12) $a_{n}$ decides $\dot{y}_{0}\left\lceil i_{n}, \ldots, \dot{y}_{n}\left\lceil i_{n}\right.\right.$ to be equal to $t_{0}^{n}, \ldots, t_{n}^{n}$, respectively,

(A13) $f_{m}^{k_{n}}\left[t_{\ell}^{n}\right] \subseteq(\varepsilon,+\infty)$ for all $\ell \leq m \leq n$.

In order to start the induction step from $n$ to $n+1$ we first find an $f_{n+1}$ in $F$ and an extension $b$ of $a_{n}$ which forces that

$$
\hat{f}_{n+1}\left(\dot{x}_{\ell}\right)<\delta \text { and } \hat{f}_{n+1}\left(\dot{y}_{\ell}\right)>\varepsilon \text { for all } \ell \leq n+1 .
$$

Then we represent $f_{n+1}$ as a limit of a sequence $\left\{f_{n+1}^{k}\right\}$ of continuous functions and find $k_{n+1}>k_{n}$ and a further extension of $b$ which forces that

$$
\hat{f}_{n+1}^{k}\left(\dot{x}_{\ell}\right)<\delta \text { and } f_{n+1}^{k}\left(\dot{y}_{\ell}\right)>\varepsilon \text { for all } \ell \leq n+1 \text { and } k \geq k_{n+1} \text {. }
$$

Now we find large enough $i>i_{n}$ and a further extension of $b$ forcing that

$$
\hat{f}_{m}^{k_{n+1}}\left[\dot { x } _ { \ell } \lceil i ] \subseteq ( - \infty , \delta ) \text { and } \hat { f } _ { m } ^ { k _ { n + 1 } } \left[\dot{y}_{\ell}\lceil i] \subseteq(\varepsilon,+\infty) \text { for all } \ell \leq m \leq n+1\right.\right. \text {. }
$$

It is now easy to make the further extensions and find $a_{n+1}, T(n+1)$ and $i_{n+1} \geq i$ satisfying the conditions (A7)-(A13) for $n+1$ in place of $n$. This completes our description of the construction.

Note that for a fixed $\ell$, for all $n \geq \ell$ the decisions $s_{\ell}^{n}$ of $a_{n}$ about the restriction $\dot{x}_{\ell} \uparrow i_{n}$ end-extend each other so we can let $u_{\ell} \in \mathbb{N}^{\mathbb{N}}$ denote their limit. Then by (A10) and (A11) we have that

$$
f_{m}^{k_{n}}\left(u_{\ell}\right)<\delta \text { for all } n \geq m \geq \ell .
$$

Similarly for a fixed $\ell$, for all $n \geq \ell$ the decisions $t_{\ell}^{n}$ of $a_{n}$ about $\dot{y}_{\ell}\left\lceil i_{n}\right.$ end-extend each other, so they converge to some $v_{\ell} \in \mathbb{N}^{\mathbb{N}}$ and (A12) and (A13) give us

$$
f_{m}^{k_{n}}\left(v_{\ell}\right)>\varepsilon \text { and } n \geq m \geq \ell .
$$

Now, for a fixed $m \geq \ell$, the sequences $\left\{f_{m}^{k_{n}}\left(u_{\ell}\right)\right\}_{n=0}^{\infty}$ and $\left\{f_{m}^{k_{n}}\left(v_{\ell}\right)\right\}_{n=0}^{\infty}$ converge to $f_{m}\left(u_{\ell}\right)$ and $f_{m}\left(v_{\ell}\right)$, respectively, so (A17) and (A18) lead us to

$$
f_{m}\left(u_{\ell}\right) \leq \delta \text { and } f_{m}\left(v_{\ell}\right) \geq \varepsilon \text { for all } m \geq \ell .
$$

Let $Q$ be the perfect subset of $\mathbb{N}^{\mathbb{N}}$ determined by the sequence $\{T(n)\}$ of levels, i.e.,

$$
Q=\left\{x \in \mathbb{N}^{\mathbb{N}}: x\left\lceil i_{n} \in T(n) \text { for all } n\right\} .\right.
$$

(The fact that $Q$ is indeed perfect follows from (A7).) Note that by (A9) both sets

$$
D=\left\{u_{\ell}: \ell \in \mathbb{N}\right\} \text { and } E=\left\{v_{\ell}: \ell \in \mathbb{N}\right\}
$$

are dense in $Q$. Since $F$ is a relatively compact set of Baire class- 1 functions, we can apply a theorem of Rosenthal [R2] (see also $\S \mathrm{C}$ below) and select a subsequence $\left\{f_{m_{k}}\right\}$ of $\left\{f_{m}\right\}$ which pointwise converges to a Baire class-1 function $f$. By (A19) we have that

$$
f(x) \leq \delta \text { and } f(y) \geq \varepsilon \text { for all } x \in D \text { and } y \in E .
$$

Since $D$ and $E$ are both dense in the perfect set $Q$, it follows that the restriction $f \uparrow Q$ has no point of continuity, contradicting the Baire characterization theorem. This finishes the proof of Lemma 1. 
In order to illustrate the potential usefulness of Lemma 1 we shall give a proof of Bourgain's theorem (Theorem 8 from the Introduction) asserting that every compact set of the first Baire class contains a dense set of $G_{\delta}$ points. Clearly, it suffices to prove only that every compact set $K$ of the first Baire class contains at least one $G_{\delta}$ point since from this it will easily follow that for every such $K$, the set of all $G_{\delta}$ points of $K$ is a set everywhere of second category in $K$. Let $\mathcal{B}$ be the standard forcing notion which forces $\mathrm{CH}$ and does not add any new reals or countable sequences of reals (see $[\mathrm{S}]$ ). By Lemma $1, K$ remains relatively compact in $V^{(\mathcal{B})}$. Since $V^{(\mathcal{B})}$ has no more reals nor Baire class-1 functions, the closure of $K$ (in $V^{(\mathcal{B})}$ ) must in fact be equal to $K$, i.e., $K$ remains compact. But in $V^{(\mathcal{B})}$ its size is at most $\aleph_{1}$ so by a classical result of Cech and Pospišil [CP], $K$ must contain a $G_{\delta}$ point, i.e., a function $f$ determined by its restriction to a countable subset $D$ of $\mathbb{N}^{\mathbb{N}}$. Since our forcing notion can decide both the function $f$ and the countable set $D$, we are done.

A similar forcing argument can be used to prove Theorem 2 (also due to J. Bourgain, see [Ta, 14-2-2]). To see this, let $K$ be a given compact set of the first Baire class and let $\mu$ be a regular Radon measure on $K$. We need to show that the Banach space $L^{1}(K, \mu)$ is separable, or equivalently that the measure algebra associated to $\mu$ is separable. Otherwise, we can find an uncountable family $G_{\alpha}(\alpha \in \Omega)$ of stochastically independent subsets of $K$ of measure $1 / 2$. For $\alpha \in \Omega$ choose compact sets $F_{\alpha}^{0} \subseteq G_{\alpha}$ and $F_{\alpha}^{1} \subseteq K \backslash G_{\alpha}$ such that

$$
\mu\left(F_{\alpha}^{0}\right)+\mu\left(F_{\alpha}^{1}\right)>\frac{1}{2} .
$$

By a result of Fremlin $[\mathrm{F}]$ there is a forcing notion $\mathcal{B}$ which forces an uncountable subset $\Omega_{0} \subseteq \Omega$ such that

$$
\mu\left(\bigcap_{\alpha \in A} F_{\alpha}^{0} \cap \bigcap_{\beta \in B} F_{\beta}^{1}\right)>0
$$

for every two disjoint finite sets $A, B \subseteq \Omega_{0}$. By Lemma 1 , in $V^{(\mathcal{B})}$, the set of extensions $\hat{K}$ is a relatively compact subset of $B_{1}\left(\mathbb{N}^{\mathbb{N}}\right)$ so its closure $\overline{\hat{K}}$ is a compact set of the first Baire class. Moreover, in $V^{(\mathcal{B})}$, the sequence $\left(\overline{\hat{F}_{\alpha}^{0}}, \overline{\hat{F}_{\alpha}^{1}}\right)\left(\alpha \in \Omega_{0}\right)$ of closures is independent in the sense that

$$
\bigcap_{\alpha \in A} \overline{\hat{F}_{1}^{0}} \cap \bigcap_{\beta \in B} \overline{\hat{F}_{\beta}^{1}} \neq \emptyset
$$

for every two disjoint finite sets $A, B \subseteq \Omega_{0}$. It follows that in $V^{(\mathcal{B})}$ the compactum $\overline{\hat{K}}$ can be mapped continuously onto the Tychonoff cube $[0,1]^{\Omega_{0}}$ of uncountable weight. This is clearly a contradiction since $\overline{\hat{K}}$ is countably tight (see [R3, Lemma 3.9]) but $[0,1]^{\Omega_{0}}$ is not.

\section{B. Free sequences of Regular pairs}

A pair $(F, G)$ of subsets of a topological space $X$ is regular if $F$ is closed, $G$ is open and $F$ is a subset of $G$. A sequence $\left(F_{\alpha}, G_{\alpha}\right)(\alpha \in \Omega)$ of regular pairs indexed by some well-ordered set $\Omega$ is free in $X$ if for every two finite subsets $A, B \subseteq \Omega$ such that every element of $A$ is smaller than every element of $B$ (in short, $A<B$ ) 
we have that

$$
\left(\bigcap_{\alpha \in A} F_{\alpha}\right) \cap\left(\bigcap_{\beta \in B}\left(X \backslash G_{\beta}\right)\right) \neq \emptyset
$$

Our interest in this notion here is based on the following fact.

Lemma 2. If $K$ is a compact set of the first Baire class, then every free sequence of regular pairs of $K$ must be countable.

Proof. Let $\left(F_{\alpha}, G_{\alpha}\right)(\alpha \in \Omega)$ be a given free sequence of regular pairs of $K$. Then by compactness for each $\beta \in \Omega$, the intersection

$$
\bigcap_{\alpha<\beta} F_{\alpha} \cap \bigcap_{\alpha \geq \beta}\left(X \backslash G_{\alpha}\right)
$$

is nonempty so we can pick an element $x_{\beta}$ from it. If $\Omega$ is uncountable, then going to an initial segment of $\Omega$, we may assume that its order type is equal to $\omega_{1}$. Again, using compactness let $x \in K$ be a complete accumulation point of $x_{\alpha}(\alpha \in \Omega)$. By another result of Rosenthal ([R3, Lemma 3.9]) there is a countable subset $\Omega_{0} \subseteq \Omega$ such that $x_{\beta}\left(\beta \in \Omega_{0}\right)$ has $x$ in its closure. Choose $\alpha \in \Omega$ such that $\alpha>\beta$ for all $\beta \in \Omega_{0}$. Then by the choice of $x_{\beta}$ 's above we have that

$$
x_{\beta} \in X \backslash G_{\alpha} \quad \text { for all } \beta \in \Omega_{0} .
$$

It follows that $x \in X \backslash G_{\alpha}$. Using again the way $x_{\beta}$ 's were chosen, we conclude that

$$
x_{\gamma} \in F_{\alpha} \text { for all } \gamma>\alpha \text {, }
$$

so $X \backslash F_{\alpha}$ is an open neighborhood of $x$ containing only countably many elements of the sequence $x_{\beta}(\beta \in \Omega)$, a contradiction. This finishes the proof of Lemma 2 .

Since we are working with subspaces of the Tychonoff cube, it will be convenient to restrict our attention to a smaller class of regular pairs of a given compact set $K$ of the first Baire class. A regular pair $(F, G)$ of $K$ is called basic if it has the form

$$
\begin{aligned}
& F=\left\{f \in K: p_{i} \leq f\left(x_{i}\right) \leq q_{i} \quad \text { for all } \quad 1 \leq i \leq n\right\} \\
& G=\left\{f \in K: r_{i}<f\left(x_{i}\right)<s_{i} \quad \text { for all } \quad 1 \leq i \leq n\right\}
\end{aligned}
$$

for some finite sequence $x_{i}(1 \leq i \leq n)$ of elements of $\mathbb{N}^{\mathbb{N}}$ and sequences

$$
r_{i}<p_{i}<q_{i}<s_{i} \quad(1 \leq i \leq n)
$$

of rationals. We are now ready to state the key lemma of this section.

Lemma 3. For every compact set $K$ of the first Baire class there is a sequence $\left(F_{\alpha}, G_{\alpha}\right)(\alpha \in \Pi)$ of basic regular pairs of $K$ indexed by some well-ordered set $\Pi$ such that the interiors of the $F_{\alpha}$ 's form a $\pi$-basis of $K$ and such that for every subset $\Omega$ of $\Pi$ the subsequence $\left(F_{\alpha}, G_{\alpha}\right)(\alpha \in \Omega)$ is free in $K$ iff the corresponding sequence $F_{\alpha}(\alpha \in \Omega)$ has the finite intersection property.

Proof. This lemma appears as Lemma 1 of our paper [To2] for an arbitrary compact space $X$ but with regular pairs rather than basic regular pairs in its conclusion. Although our additional requirement here makes no difference in the proof, for the convenience of the reader we reproduce the argument from [To2]. Let $\pi$ be the minimal size of a $\pi$-basis of $K$, i.e., a family $\mathcal{P}$ of nonempty open sets of $K$ such that every nonempty open subset of $K$ includes an element of $\mathcal{P}$. Working separately below each member of a maximal disjoint family of open subsets of $K$ 
we may assume that the $\pi$-weight of $K$ below every nonempty open subset of $K$ is again equal to $\pi$. Let $U_{\alpha}(\alpha<\pi)$ be a fixed $\pi$-basis of $K$. The desired sequence $\left(F_{\alpha}, G_{\alpha}\right)(\alpha<\pi)$ of basic regular pairs of $K$ is constructed recursively on $\alpha$ with the general requirement that

$$
\emptyset \neq \operatorname{int}\left(F_{\alpha}\right) \subseteq F_{\alpha} \subseteq G_{\alpha} \subseteq U_{\alpha} .
$$

Let $\beta<\pi$ be given and suppose $\left(F_{\alpha}, G_{\alpha}\right)$ has been chosen for all $\alpha<\beta$. Let $\mathcal{F}_{\beta}$ be the family of all nonempty finite intersections of sets of the form $F_{\alpha}$ or $K \backslash G_{\alpha}$ where $\alpha<\beta$. Since every member of $\mathcal{F}_{\beta}$ is a compact $G_{\delta}$ set and since $\mathcal{F}_{\beta}$ has size $<\pi$, by compactness and by our assumption about the $\pi$-weight below $U_{\beta}$, there is a nonempty open subset $V_{\beta}$ of $U_{\beta}$ which includes no member of $\mathcal{F}_{\beta}$. Choose an arbitrary basic regular pair $\left(F_{\beta}, G_{\beta}\right)$ such that

$$
\emptyset \neq \operatorname{int}\left(F_{\beta}\right) \subseteq F_{\beta} \subseteq G_{\beta} \subseteq V_{\beta} .
$$

This completes the construction. Suppose $\Omega$ is a subset of $\pi$ such that $F_{\alpha}(\alpha \in \Omega)$ is centered. Let $A<B$ be two finite subsets of $\Omega$. The proof that

$$
\bigcap_{\alpha \in A} F_{\alpha} \cap \bigcap_{\beta \in B}\left(K \backslash G_{\beta}\right) \neq \emptyset
$$

is by induction on the size of $B$. Note that $B=\emptyset$ reduces to our assumption that $F_{\alpha}(\alpha \in \Omega)$ is centered, so suppose $n=|B|>0$ and that (B7) holds for all pairs $\left(A, B_{0}\right)$ with $B_{0}$ of size $<n$. Let $\beta=\max (B)$ and let $B_{0}=B \backslash\{\beta\}$. Let

$$
F=\bigcap_{\alpha \in A} F_{\alpha} \cap \bigcap_{\beta \in B_{0}}\left(K \backslash G_{\beta}\right) .
$$

Then $F$ is nonempty and is a member of the family $\mathcal{F}_{\beta}$. By (B6) and our choice of $V_{\beta}$ the set $F$ is not included in $G_{\beta}$, which is exactly the statement of (B7) that we are trying to prove. This completes the proof.

Remark 1. The proper context of Lemma 3 is really the class of all compact Hausdorff spaces and the reader is referred to [To2] for a more appropriate explanation of this result than can be given here.

As a first approximation to the proof of Theorem 1 we now show that every compact set of the first Baire class has some properties shared by all metric spaces.

Lemma 4. Every set $K$ of the first Baire class has a $\sigma$-disjoint $\pi$-basis.

Proof. Let $\left(F_{\alpha}, G_{\alpha}\right)(\alpha \in \Pi)$ be a sequence of basic regular pairs of $K$ given by Lemma 3 . Let $\mathcal{B}$ be the algebra of all regular-open subsets of $K$ which we consider as a forcing notion. Let $\dot{\mathcal{G}}$ be the generic filter of $\mathcal{B}$. In $V^{(\mathcal{B})}$, let

$$
\dot{\Omega}=\left\{\beta \in \Pi: \operatorname{int}\left(F_{\beta}\right) \in \dot{\mathcal{G}}\right\} .
$$

Claim 1. It is forced by $\mathcal{B}$ that $\dot{\Omega}$ is countable.

Proof. By Lemma 1, in $V^{(\mathcal{B})}$,

$$
\hat{K}=\{\hat{f}: f \in K\}
$$

is a relatively compact subset of Baire class- 1 functions, i.e., its pointwise closure $K_{\mathcal{B}}$ inside the Tychonoff cube consists only of Baire class-1 functions. For every 
basic regular pair $(F, G)$ given by the definitions (B3) and (B4) above, we can use the same definitions in $V^{(\mathcal{B})}$ and get the regular pair

$$
\begin{aligned}
& F_{\mathcal{B}}=\left\{f \in K_{\mathcal{B}}: \quad p_{i} \leq f\left(x_{i}\right) \leq q_{i} \quad \text { for } \quad 1 \leq i \leq n\right\}, \\
& G_{\mathcal{B}}=\left\{f \in K_{\mathcal{B}}: \quad r_{i}<f\left(x_{i}\right)<s_{i} \quad \text { for } \quad 1 \leq i \leq n\right\}
\end{aligned}
$$

of the space $K_{\mathcal{B}}$. It is also easily checked that the sequence $\left(\left(F_{\alpha}\right)_{\mathcal{B}},\left(G_{\alpha}\right)_{\mathcal{B}}\right)(\alpha \in \Pi)$ of extensions still satisfies the second conclusion of Lemma 3 . This is an immediate consequence of the fact that for every $\alpha \in \Pi$,

$$
\left(F_{\alpha}\right)_{\mathcal{B}} \cap \hat{K}=\hat{F}_{\alpha} \quad \text { and } \quad\left(G_{\alpha}\right)_{\mathcal{B}} \cap \hat{K}=\hat{G}_{\alpha}
$$

and the fact that the conclusion concerns finite subsets of $\Pi$ which can be decided by conditions of $\mathcal{B}^{+}$. It follows that in $V^{(\mathcal{B})}$ the sequence $\left(\left(F_{\alpha}\right)_{\mathcal{B}},\left(G_{\alpha}\right)_{\mathcal{B}}\right)(\alpha \in \dot{\Omega})$ is a free sequence of regular pairs of the compactum $K_{\mathcal{B}}$ of the first Baire class. By Lemma 2 we conclude that $\dot{\Omega}$ must be countable. This proves the Claim.

By the first conclusion of Lemma $3,\left\{\operatorname{int}\left(F_{\beta}\right): \beta \in \dot{\Omega}\right\}$ is a subset of $\dot{\mathcal{G}}$ which generates $\dot{\mathcal{G}}$, i.e., every element of $\dot{\mathcal{G}}$ includes some $\operatorname{int}\left(F_{\beta}\right)$ for $\beta \in \dot{\Omega}$. It follows that we can find a $\mathcal{B}$-name $\tau$ for a function from $\mathbb{N}$ into $\dot{\mathcal{G}}$ such that every condition of $\mathcal{B}^{+}$forces that

(B11) $\tau(m) \supseteq \tau(n)$ for $m<n$,

(B12) for every $O \in \dot{\mathcal{G}}$ there is $n$ such that $\tau(n) \subseteq O$.

Build a sequence $\mathcal{P}_{n}(n \in \mathbb{N})$ of maximal antichains of $\mathcal{B}^{+}$such that for all $n$

(B13) $\mathcal{P}_{n+1}$ refines $\mathcal{P}_{n}$,

(B14) for every $O \in \mathcal{P}_{n}$ there is $G(O) \in \mathcal{B}^{+}$such that $O$ forces $\tau(n)=G(O)$.

Claim 2. $\mathcal{P}=\bigcup_{n=1}^{\infty} \mathcal{P}_{n}$ is a $\pi$-basis of $K$.

Proof. It suffices to show that every $U \in \mathcal{B}^{+}$includes some member of $\mathcal{P}$. Since $U$ forces itself to be a member of the generic filter $\dot{\mathcal{G}}$, by (B12), we can find $n \in \mathbb{N}$, $V \subseteq U$ and $W \in \mathcal{B}^{+}$such that $W$ forces $\tau(n)=V$. Since $\mathcal{P}_{n}$ is a maximal antichain of $\mathcal{B}^{+}$, there is $O \in \mathcal{P}_{n}$ such that $W \cap O$ is nonempty. It follows by (B14) that $W \cap O$ forces that

$$
V=\tau(n)=G(O) .
$$

Thus we must have that $G(O)=V$. Since $V \subseteq U$, it follows that $O$ forces that $U$ is a member of the generic filter $\dot{\mathcal{G}}$. Since $\mathcal{B}^{+}$is a separative forcing notion, this can happen only when $O$ is a subset of $U$. This completes the proof of Claim 2 and also the proof of Lemma 4 .

Now we are ready to finish the proof of Theorem 1. Note that if $K$ has a $\sigma$ disjoint $\pi$-basis, then so does every dense subspace of $K$. By Bourgain's theorem (Theorem 8 above) $K$ contains a dense first countable subspace. So to finish the proof of Theorem 1 all we need is to apply the following result of H.E. White, Jr. [W, Corollary 2.2], whose proof is given here for completeness.

Lemma 5. Every first countable Hausdorff space $X$ with a $\sigma$-disjoint $\pi$-base has a dense metrizable subspace. 
Proof. For each $x \in X$, let $\left\{O_{n}(x)\right\}$ be a fixed decreasing sequence of open neighborhoods of $x$ forming a local basis of $x$ in $X$. For a set $D \subseteq X$ and a function $\phi: D \rightarrow \mathbb{N}$, let $\mathcal{O}_{\phi}(D)$ denote the collection $O_{\phi(x)}(x)(x \in D)$ of open subsets of $X$. Let $\mathcal{P}=\bigcup_{i=1}^{\infty} \mathcal{P}_{i}$ be a fixed $\sigma$-disjoint $\pi$-basis of $X$. By induction on $i$ we build a sequence $\phi_{i}: D_{i} \rightarrow \mathbb{N}$ of mappings from subsets $D_{i}$ of $X$ such that

(B16) $D_{i} \subseteq D_{i+1}$,

(B17) $\mathcal{O}_{\phi_{i}}\left(D_{i}\right)$ is a disjoint collection and its union is dense in $X$,

(B18) $\mathcal{O}_{\phi_{i+1}}\left(D_{i+1}\right)$ refines $\mathcal{O}_{\phi_{i}}\left(D_{i}\right)$,

(B19) $\mathcal{O}_{\phi_{i+1}}\left(D_{i+1} \backslash D_{i}\right)$ refines $\mathcal{P}_{i+1}\left(\right.$ where $\left.D_{0}=\emptyset\right)$,

(B20) $\phi_{i+1}(x)>\phi_{i}(x)$ for all $x \in D_{i}$.

There are no difficulties in constructing these objects. For if we are given $\phi_{i}$ : $D_{i} \rightarrow \mathbb{N}$, for each $x \in D_{i}$, let $\phi_{i+1}(x)=n+1$ where $n=\phi_{i}(x)$. If $R_{i}(x)=$ $O_{n}(x) \backslash \overline{O_{n+1}(x)}$ is nonempty we choose a subset $D_{i+1}(x)$ of the intersection

$$
R_{i}(x) \cap\left(\bigcup \mathcal{P}_{i+1}\right)
$$

and a function $\psi_{x}: D_{i+1}(x) \rightarrow \mathbb{N}$ such that $\mathcal{O}_{\psi_{x}}\left(D_{i+1}(x)\right)$ is a disjoint collection which refines $\mathcal{P}_{i+1}$ and whose union is dense in $R_{i}(x)$. Finally, let $\phi_{i+1}$ be the union of $\phi_{i+1}\left\lceil D_{i}\right.$ defined above and the functions $\psi_{x}\left(x \in D_{i}, R_{i}(x) \neq \emptyset\right)$. Having done this, we let $D=\bigcup_{i=1}^{\infty} D_{i}$ and observe that $D$ is dense in $X$ as it intersects every element of the $\pi$-basis $\mathcal{P}$. By (B17), (B18) and (B20) we conclude that the restriction of

$$
\bigcup_{i=1}^{\infty} \mathcal{O}_{\phi_{i}}\left(D_{i}\right)
$$

to $D$ is a $\sigma$-discrete basis of $D$. This completes the proof.

Remark 2. One of the reasons the proof of Lemma 5 has been reproduced here is to expose a property of the dense metrizable subspace given by it. Namely, note that if $X$ is dense-in-itself, the metrizable subspace $D$ given by the proof of Lemma 5 is $\sigma$-discrete and therefore meager. This is not an accident, for if we let $K$ be the split interval $S(I)$ described in the introduction we see that every metrizable subspace of $S(I)$ is indeed meager. In fact, it is not hard to see that every metrizable subspace of $S(I)$ is actually countable. This is in striking contrast with the other standard classes of compacta occurring in functional analysis, for in any instance where the analogue of Theorem 1 was proved, the proof actually produces a dense $G_{\delta}$ metrizable subspace. Perhaps this can be used as some explanation of why the Namioka-style arguments about joint versus separate continuity used so efficiently in those other cases (see $[\mathrm{Na}],[\mathrm{MN}]$ ) cannot be used for the first Baire class. There is another example of a compactum $K$ of the first Baire class without a dense $G_{\delta}$ metrizable subspace found in the literature. This is the example of Argyros and Mercourakis [AM, Theorem 3.6] used for the purpose of analyzing various questions concerning differentiability of norms in Banach spaces. We note that Argyros and Mercourakis have left open the question (to which we now know the answer) of whether their space contains a dense metrizable subspace (see [MN, 5.8(1)]).

\section{Parameterized sequential compactness}

In this section we prove a version of Rosenthal's theorem about sequential compactness of compact sets of Baire class-1 functions ([R2], [R3]). This will be done as 
an application of a parameterized perfect-set property of analytic sets, a theorem which at the same time incorporates the classical perfect-set property of analytic sets of M. Souslin and the Ramsey property of analytic sets of Nash-Williams, Galvin-Prikry, and Silver (see [Ke]). The family of infinite subsets of $\mathbb{N}$ is naturally identified with a subset of the Cantor cube $2^{\mathbb{N}}$ from where it takes its topology. It is therefore natural to use $2_{\infty}^{\mathbb{N}}$ to denote the family of all infinite subsets of $\mathbb{N}$ in this topological context.

Theorem 10 (Parameterized perfect-set theorem). For every analytic subset $A$ of $2^{\mathbb{N}} \times 2_{\infty}^{\mathbb{N}}$ there is a perfect set $P \subseteq 2^{\mathbb{N}}$ and there is an infinite set $M \subseteq \mathbb{N}$ such that $P \times 2_{\infty}^{M}$ is either disjoint from or included in $A$.

The reader is referred to two proofs of this result, the one which uses forcing due to A. Miller [Mi], and the other which is more classical due to J. Pawlikowski [Pa].

Theorem 11. Let $f_{s}\left(s \in 2^{<\mathbb{N}}\right)$ be a relatively compact subset of Baire class-1 functions defined on some Polish space $X$. Then there is a perfect set $P \subseteq 2^{\mathbb{N}}$ and an infinite strictly increasing sequence $\left\{n_{k}\right\}$ of integers such that $\left\{f_{a \uparrow n_{k}}\right\}$ is pointwise convergent for every $a \in P$.

Proof. Let

$$
A=\left\{(a, M) \in 2^{\mathbb{N}} \times 2_{\infty}^{\mathbb{N}}:\left\{f_{a \nmid m}\right\}_{m \in M} \text { is pointwise convergent on } X\right\} .
$$

Note that $A$ is a coanalytic subset of the product so Theorem 10 applies to it. Hence we can find a perfect set $P \subseteq 2^{\mathbb{N}}$ and an infinite set $M \subseteq \mathbb{N}$ such that $P \times 2_{\infty}^{M}$ is either disjoint from or included in $A$. The second alternative is what we want so let us show that the first one is impossible. For this it suffices to show that for some $a \in P$ the sequence $\left\{f_{a \uparrow m}\right\}_{m \in M}$ has an infinite pointwise-convergent subsequence. This of course follows from the main result of [R2], but for completeness we give a proof of this fact here. For notational simplicity we assume $M=\mathbb{N}$ and write $g_{n}$ instead of $f_{a \nmid n}$. Suppose $\left\{g_{n}\right\}$ contains no infinite convergent subsequence. Then a straightforward diagonal argument shows that there is an infinite set of integers $N$ and two rationals $\delta<\varepsilon$ such that

$$
\begin{aligned}
& \text { for every infinite } L \subseteq N \text { there is } x \in X \text { such that } \\
& \qquad\left\{n \in L: g_{n}(x) \leq \delta\right\} \text { and }\left\{n \in L: g_{n}(x) \geq \varepsilon\right\} \text { are both infinite. }
\end{aligned}
$$

For $L \in 2_{\infty}^{N}$ let $L_{\text {even }}$ (resp., $L_{\text {odd }}$ ) be the set of all $n \in L$ that occupy even (resp., odd) places in the increasing enumeration of $L$. Let $B$ be the set of all $L \in 2_{\infty}^{N}$ for which there is $x \in X$ such that

$$
g_{n}(x) \leq \delta \text { for all } n \in L_{\text {even }} \text { and } g_{n}(x) \geq \varepsilon \text { for all } n \in L_{\text {odd }} .
$$

Clearly, $B$ is an analytic subset of $2_{\infty}^{N}$, so by the nonparameterized version of Theorem 10 (a theorem of Silver) there is $M \in 2_{\infty}^{N}$ such that $2_{\infty}^{M}$ is either disjoint from or included in $B$. Note that by $(\mathrm{C} 1)$ the first alternative is impossible, so we are left with the alternative $2_{\infty}^{M} \subseteq B$. Let $\left\{m_{k}\right\}$ be the increasing enumeration of $M_{\text {even }}$. Refining the sequence, if necessary, we assume that $\left\{g_{m_{k}}\right\}$ is a relatively discrete subset of $B_{1}\left(\mathbb{N}^{\mathbb{N}}\right)$. By our assumption $\left\{g_{m_{k}}\right\}$ is a relatively compact subset of $B_{1}\left(\mathbb{N}^{\mathbb{N}}\right)$, so the mapping $k \mapsto g_{m_{k}}$ extends to a continuous mapping $\mathcal{U} \mapsto g_{\mathcal{U}}$ of $\beta \mathbb{N}$ into $B_{1}\left(\mathbb{N}^{\mathbb{N}}\right)$ given by

$$
g_{\mathcal{U}}(x)=\lim _{k \rightarrow \mathcal{U}} g_{m_{k}}(x)
$$


The following fact gives us the desired contradiction that finishes the proof of Theorem 11.

Claim 1. $g_{\mathcal{U}} \neq g_{\mathcal{V}}$ for $\mathcal{U} \neq \mathcal{V}$.

Proof. Clearly, we may assume that $\mathcal{U}$ and $\mathcal{V}$ are nonprincipal ultrafilters. Choose $L_{0} \in \mathcal{U}$ and $L_{1} \in \mathcal{V}$ such that $L_{0} \cap L_{1}=\emptyset$. Using some points from $M_{\text {odd }}$, we can build an infinite $L \subseteq M$ such that $L_{0} \subseteq L_{\text {odd }}$ and $L_{1} \subseteq L_{\text {even }}$. Since $L \in B$, there is $x \in X$ such that $(\mathrm{C} 2)$ holds. It follows that

$$
g_{\mathcal{U}}(x) \leq \delta<\varepsilon \leq g_{\mathcal{V}}(x) .
$$

This finishes the proof.

The proof of Theorem 11 shows that we can also have the following form of a parameterized Rosenthal's $\ell^{1}$-theorem which is slightly stronger than the corresponding result of J. Stern [Ste].

Theorem 12. Let $x_{s}\left(s \in 2^{<\mathbb{N}}\right)$ be a bounded sequence of elements of some Banach space. Then there is a perfect set $P \subseteq 2^{\mathbb{N}}$ and an infinite sequence $\left\{n_{k}\right\}$ of integers such that

(a) $\left\{x_{a \nmid n_{k}}\right\}$ is weakly-Cauchy for all $a \in P$, or

(b) there is $\delta>0$ such that for every $a \in P$ and every finite sequence $\lambda_{1}, \ldots, \lambda_{m}$ of scalars,

$$
\left\|\sum_{k=1}^{m} \lambda_{k} x_{a \uparrow n_{k}}\right\| \geq \delta \cdot \sum_{k=1}^{m}\left|\lambda_{k}\right| .
$$

The parameterized perfect-set property admits extensions to all finite dimensions. Particularly simple and to us useful is the 2 -dimensional version. To state this property, for a set $P$ let $P^{[2]}$ denote its deleted square, i.e., the set

$$
P^{[2]}=\{(x, y) \in P: x \neq y\} .
$$

A subset $B$ of $X^{2} \times 2_{\infty}^{\mathbb{N}}$ is symmetric if it is symmetric with respect to the first two coordinates, i.e., for every $(x, y, z) \in X^{2} \times 2^{\mathbb{N}}$,

$$
(x, y, z) \in B \quad \text { if and only if }(y, x, z) \in B .
$$

We call $B \subseteq X^{2} \times 2_{\infty}^{\mathbb{N}}$ invariant if it is invariant under finite changes of the third coordinate, or more precisely,

$$
\begin{aligned}
& (x, y, z) \in B \text { is equivalent to }\left(x, y, z^{\prime}\right) \in B \\
& \text { whenever the symmetric difference of } z \text { and } z^{\prime} \text { is finite. }
\end{aligned}
$$

Theorem 13. Let $X$ be a Polish space with no isolated points and let $A$ be an analytic, symmetric, and invariant subset of the product $X^{2} \times 2_{\infty}^{\mathbb{N}}$. Then there exist a perfect set $P \subseteq X$ and an infinite set $M \subseteq \mathbb{N}$ such that $P^{[2]} \times 2_{\infty}^{M}$ is either disjoint from or included in $A$.

Every application of this result that we have here in this paper can be more transparently seen as a use first of Theorem 10 and then a use of a much simpler form of Theorem 13, its nonparameterized version, originally due to F. Galvin (see $[\mathrm{Bl}])$ : 
Theorem 14. For every perfect Polish space $X$ and every symmetric analytic relation $A \subseteq X^{2}$ there is a perfect set $P \subseteq X$ such that $P^{[2]}$ is either disjoint from or included in $A$.

We refer the reader to the papers of Blass [Bl], Miller [Mi] and Pawlikowski [Pa] from where proofs of these results (as well as their generalizations to all finite dimensions) can be extracted. In $\S \mathrm{G}$ below we shall need an infinite-dimensional version of Theorem 14 .

\section{Premetric Compacta of Degree 2}

We are now ready to start the proof of Theorem 3 as well as the proof of Theorem 5 . So let $K$ be a given compactum which consists of Baire class- 1 functions defined on the Baire space $\mathbb{N}^{\mathbb{N}}$. If $K$ contains no uncountable discrete subspace, then by Theorem 1 it is (hereditarily) separable. So to prove both theorems it suffices to assume that $K$ is separable and prove that $K$ is an at most two-to-one preimage of a compact metric space unless it contains a discrete subspace of size continuum. Let $K_{0}$ be a fixed countable dense subset of $K$. Increasing the topology of $\mathbb{N}^{\mathbb{N}}$ a bit but not changing its topological type we may assume that the countable set $K_{0}$ consists of continuous functions on the Baire space $\mathbb{N}^{\mathbb{N}}$ (see [Ke, $\left.\S 13 \mathrm{~A}\right]$ ).

The basic open sets of $\mathbb{N}^{\mathbb{N}}$ are naturally coded by finite sequences of elements of $\mathbb{N}$. The basic open set determined by some $t \in \mathbb{N}<\mathbb{N}$ is denoted by $[t]$. Note that $\mathbb{N}<\mathbb{N}$ is a tree under end-extension. A subtree of $\mathbb{N}<\mathbb{N}$ will always be assumed to be downwards closed. For a subtree $T$ of $\mathbb{N}<\mathbb{N}$, we let $[T]$ denote the set of all elements of $\mathbb{N}^{\mathbb{N}}$ determined by the infinite branches of $T$. We shall also consider subtrees of $(\mathbb{N}<\mathbb{N})^{2},(\mathbb{N}<\mathbb{N})^{3}$, etc. In any such case we implicitly assume that such a subtree $T$ consists of $k$-tuples whose entries have equal lengths (and of course that $T$ is downward closed). Again, for such a subtree $T \subseteq\left(\mathbb{N}^{<N}\right)^{k}$, we let $[T]$ denote the subset of $\left(\mathbb{N}^{\mathbb{N}}\right)^{k}$ determined by the infinite branches of $T$. A subtree $T$ of some power $\left(\mathbb{N}^{<\mathbb{N}}\right)^{k}$ is perfect if

$$
\begin{array}{r}
\text { every }\left(s^{0}, s^{1}, \ldots, s^{k-1}\right) \text { from } T \text { has two extensions }\left(t^{0}, t^{1}, \ldots, t^{k-1}\right) \\
\text { and }\left(u^{0}, u^{1}, \ldots, u^{k-1}\right) \text { in } T \text { which properly split in the sense that }
\end{array}
$$

they belong to the same level of $T$ and that $t^{i} \neq u^{i}$ for all $i<k$.

Note that if the dichotomy of Theorem 5 fails for our space $K$, or in other words, if $K$ is not an at most two-to-one preimage of a compact metric space, then for every countable subset $D$ of $\mathbb{N}^{\mathbb{N}}$ there exist three distinct elements $f^{0}, f^{1}, f^{2}$ of $K$ which agree on $D$, i.e., $f^{0} \uparrow D=f^{1} \uparrow D=f^{2} \uparrow D$. Thus a simple recursion on countable ordinals would give us a sequence $f_{\alpha}^{i}\left(\alpha<\omega_{1}, i<3\right)$ of triples of elements of $K$ and a sequence $x_{\alpha}^{i}\left(\alpha<\omega_{1}, i<3\right)$ of triples of elements of $\mathbb{N}^{\mathbb{N}}$ such that:

(D2) $f_{\beta}^{i}\left(x_{\alpha}^{k}\right)=f_{\beta}^{j}\left(x_{\alpha}^{k}\right)$ for all $\alpha<\beta$ and $i, j, k<3$,

(D3) $f_{\alpha}^{0}\left(x_{\alpha}^{0}\right) \neq f_{\alpha}^{1}\left(x_{\alpha}^{0}\right), f_{\alpha}^{1}\left(x_{\alpha}^{1}\right) \neq f_{\alpha}^{2}\left(x_{\alpha}^{1}\right)$, and $f_{\alpha}^{0}\left(x_{\alpha}^{2}\right) \neq f_{\alpha}^{2}\left(x_{\alpha}^{2}\right)$.

Refining, if necessary, we may assume that there is a sequence $I^{i j}(i, j<3)$ of open rational intervals such that

(D4) $f_{\alpha}^{i}\left(x_{\alpha}^{j}\right) \in I^{i j}$ for all $\alpha<\omega_{1}$ and $i, j<3$,

(D5) $\overline{I^{00}} \cap \overline{I^{10}}=\overline{I^{11}} \cap \overline{I^{21}}=\overline{I^{02}} \cap \overline{I^{22}}=\emptyset$.

Note that by (D2) and (D3), if $\alpha<\beta$, then the two triples $\left(x_{\alpha}^{0}, x_{\alpha}^{1}, x_{\alpha}^{2}\right)$ and $\left(x_{\beta}^{0}, x_{\beta}^{1}, x_{\beta}^{2}\right)$ have no points in common. So applying the standard Cantor-Bendixson 
analysis to the subtree of $\left(\mathbb{N}^{<\mathbb{N}}\right)^{3}$ generated by $\left(x_{\alpha}^{0}, x_{\alpha}^{1}, x_{\alpha}^{2}\right)\left(\alpha<\omega_{1}\right)$ we obtain a perfect subtree $T$ of $\left(\mathbb{N}^{<\mathbb{N}}\right)^{3}$ such that

(D6) for every $\left(t^{0}, t^{1}, t^{2}\right) \in T$ there exist uncountably many $\alpha$ 's such that

$$
\left(x_{\alpha}^{0}, x_{\alpha}^{1}, x_{\alpha}^{2}\right) \in[T] \text { and } t^{i} \subseteq x_{\alpha}^{i} \text { for all } i<3 .
$$

By recursion on the complete binary tree $2^{<\mathbb{N}}$ of finite sequences of 0 's and 1 's we build

$$
\begin{aligned}
\left(g_{\sigma}^{0}, g_{\sigma}^{1}, g_{\sigma}^{2}\right) \in K_{0}^{3} & \left(\sigma \in 2^{<\mathbb{N}}\right) \quad \text { and } \\
\left(t_{\sigma}^{0}, t_{\sigma}^{1}, t_{\sigma}^{2}\right) \in T & \left(\sigma \in 2^{<\mathbb{N}}\right)
\end{aligned}
$$

such that:

(D7) if $\tau$ extends $\sigma$, then $\left(t_{\tau}^{0}, t_{\tau}^{1}, t_{\tau}^{2}\right)$ extends $\left(t_{\sigma}^{0}, t_{\sigma}^{1}, t_{\sigma}^{2}\right)$,

(D8) $\left(t_{\sigma 0}^{0}, t_{\sigma 0}^{1}, t_{\sigma 0}^{2}\right)$ and $\left(t_{\sigma 1}^{0}, t_{\sigma 1}^{1}, t_{\sigma 1}^{2}\right)$ are two extensions of $\left(t_{\sigma}^{0}, t_{\sigma}^{1}, t_{\sigma}^{2}\right)$ which properly split,

(D9) $g_{\sigma}^{i}\left[t_{\sigma}^{j}\right] \subseteq I^{i j}$ for all $\sigma \in 2^{<\mathbb{N}}$ and $i, j<3$,

(D10) if $\sigma \neq \tau$ have the same length $n$, then for all $i, j, k<3$,

$$
\sup _{x \in\left[t_{\sigma}^{k}\right]}\left|g_{\tau}^{i}(x)-g_{\tau}^{j}(x)\right| \leq \frac{1}{2^{n}} .
$$

Since the moves needed to complete the inductive step include the moves needed for the initial step, we present only the inductive step. So suppose we have determined these objects for all $\sigma$ of length $\leq n$. The $\sigma$ of length $n$ will be listed according to the lexicographical order $<$ and the splitting procedure will be done successively according to this list. Thus, suppose $\sigma$ is given such that for all $\tau<\sigma$ we have determined $\left(g_{\tau \varepsilon}^{0}, g_{\tau \varepsilon}^{1}, g_{\tau \varepsilon}^{2}\right) \in K_{0}^{3}(\varepsilon<2)$ and two extensions $\left(u_{\tau 0}^{0}, u_{\tau 0}^{1}, u_{\tau 0}^{2}\right)$ and $\left(u_{\tau 1}^{0}, u_{\tau 1}^{1}, u_{\tau 1}^{2}\right)$ of $\left(t_{\tau}^{0}, t_{\tau}^{1}, t_{\tau}^{2}\right)$ in $T$ which properly split. Moreover we assume that on this stage of the successive procedure we have determined some extensions $\left(v_{\tau}^{0}, v_{\tau}^{1}, v_{\tau}^{2}\right)$ of $\left(t_{\tau}^{0}, t_{\tau}^{1}, t_{\tau}^{2}\right)$ for all $\tau \geq \sigma$ in $2^{n}$ such that

(D11) $g_{\tau \varepsilon}^{i}\left[u_{\tau \varepsilon}^{j}\right] \subseteq I^{i j} \quad$ for all $i, j<3, \tau<\sigma$ and $\varepsilon<2$,

(D12) for all $\tau<\sigma, \varepsilon<2$ and $i, j<3$,

$$
\sup _{x \in[s]}\left|g_{\tau \varepsilon}^{i}(x)-g_{\tau \varepsilon}^{j}(x)\right|<\frac{1}{2^{n+1}}
$$

for all $s \in\left\{u_{\rho \delta}^{k}: \rho<\sigma, \delta<2, k<3, \rho \delta \neq \tau \varepsilon\right\} \cup\left\{v_{\rho}^{k}: \rho \geq \sigma, k<3\right\}$.

We need to show how to split at this $\sigma$ at the price of extending the already defined $u_{\tau \varepsilon}^{i}$ 's and $v_{\rho}^{j}$ 's $(\tau<\sigma<\rho, i, j<3, \varepsilon<2)$. Since $T$ is perfect we first pick two properly splitting extensions $\left(w_{\sigma 0}^{0}, w_{\sigma 0}^{1}, w_{\sigma 0}^{2}\right)$ and $\left(w_{\sigma 1}^{0} w_{\sigma 1}^{1} w_{\sigma 1}^{2}\right)$ of $\left(v_{\sigma}^{0}, v_{\sigma}^{1}, v_{\sigma}^{2}\right)$. Find an $\alpha<\omega_{1}$ such that $\left(x_{\alpha}^{0}, x_{\alpha}^{1}, x_{\alpha}^{2}\right) \in[T]$ and it extends $\left(w_{\sigma 1}^{0}, w_{\sigma 1}^{1}, w_{\sigma 1}^{2}\right)$. For every $\tau$ in the set

$$
\Omega=\left\{\rho \varepsilon: \rho \in 2^{n}, \rho<\sigma, \varepsilon<2\right\} \cup\left\{\rho \in 2^{n}: \rho>\sigma\right\}
$$

choose $\alpha_{\tau}<\omega_{1}$ such that $\left(x_{\alpha_{\tau}}^{0}, x_{\alpha_{\tau}}^{1}, x_{\alpha_{\tau}}^{2}\right) \in[T]$ and it extends $\left(u_{\tau}^{0}, u_{\tau}^{1}, u_{\tau}^{2}\right)$ or $\left(v_{\tau}^{0}, v_{\tau}^{1}, v_{\tau}^{2}\right)$ depending on which of the two sets forming $\Omega$ the sequence $\tau$ belongs to. Again using (D6) choose a countable ordinal

$$
\beta>\max \left\{\alpha+\alpha_{\tau}: \tau \in \Omega\right\}
$$


such that $\left(x_{\beta}^{0}, x_{\beta}^{1}, x_{\beta}^{2}\right) \in[T]$ and it extends $\left(w_{\sigma 0}^{0} w_{\sigma 0}^{1} w_{\sigma 0}^{2}\right)$. Since $K_{0}$ is pointwisedense in $K$ there exist $g_{\sigma 0}^{0}, g_{\sigma 0}^{1}$ and $g_{\sigma 0}^{2}$ in $K_{0}$ approximating $f_{\beta}^{0}, f_{\beta}^{1}$ and $f_{\beta}^{2}$ as follows (see (D4) and (D2)):

(D13) $g_{\sigma 0}^{i}\left(x_{\beta}^{j}\right) \in I^{i j}$ for all $i, j<3$,

(D14) $\left|g_{\sigma 0}^{i}\left(x_{\xi}^{k}\right)-g_{\sigma 0}^{j}\left(x_{\xi}^{k}\right)\right|<2^{-n-1}$ for all $i, j, k<3$ and $\xi=\alpha$ or $\xi=\alpha_{\tau}$ for some $\tau \in \Omega$.

Since the $g_{\sigma 0}^{i}$ 's are continuous functions, we can find a sufficiently large integer $\ell$ such that

(D15) $g_{\sigma 0}^{i}\left[x_{\beta}^{j}\lceil\ell] \subseteq I^{i j} \quad\right.$ for all $i, j<3$,

(D16) for all $s \in\left\{x_{\xi}^{k} \mid \ell: k<3, \xi=\alpha\right.$ or $\xi=\alpha_{\tau}$ for some $\left.\tau \in \Omega\right\}$ and all $i, j<3$,

$$
\sup _{x \in[s]}\left|g_{\sigma 0}^{i}(x)-g_{\sigma 0}^{j}(x)\right|<\frac{1}{2^{n+1}} .
$$

This gives us $g_{\sigma 0}^{0}, g_{\sigma 0}^{1}$ and $g_{\sigma 0}^{2}$ which satisfy the requirements (D11) and (D12) at the expense of increasing

(D17) $u_{\rho \varepsilon}^{k} \quad$ to $\quad \bar{u}_{\rho \varepsilon}^{k}=x_{\alpha_{\rho \varepsilon}}\lceil\ell \quad(\rho<\sigma, \varepsilon<2, k<3)$,

(D18) $v_{\rho}^{k}$ to $\bar{v}_{\rho}^{k}=x_{\alpha_{\rho}}^{k} \mid \ell \quad(\rho>\sigma, k<3)$,

(D19) $w_{\sigma 1}^{k} \quad$ to $\quad \bar{w}_{\sigma 1}^{k}=x_{\alpha}^{k}\lceil\ell \quad(k<3)$, and

(D20) $w_{\sigma 0}^{k}$ to $\bar{w}_{\sigma 0}^{k}=x_{\beta}^{k}\lceil\ell \quad(k<3)$.

Choose now a countable ordinal $\gamma$ above

$$
\max \left\{\alpha+\beta+\alpha_{\tau}: \tau \in \Omega\right\}
$$

such that $\left(x_{\gamma}^{0}, x_{\gamma}^{1}, x_{\gamma}^{2}\right) \in[T]$ and it extends $\left(\bar{w}_{\sigma 1}^{0}, \bar{w}_{\sigma 1}^{1}, \bar{w}_{\sigma 1}^{2}\right)$. Again, since $K_{0}$ is pointwise dense in $K$, there exist $g_{\sigma 1}^{0}, g_{\sigma 1}^{1}$ and $g_{\sigma 1}^{2}$ in $K_{0}$ approximating $f_{\gamma}^{0}, f_{\gamma}^{1}$ and $f_{\gamma}^{2}$ as follows (see (D4) and (D2)):

(D21) $g_{\sigma 1}^{i}\left(x_{\gamma}^{j}\right) \in I^{i j}$ for all $i, j<3$,

(D22) $\left|g_{\sigma 1}^{i}\left(x_{\xi}^{k}\right)-g_{\sigma 1}^{j}\left(x_{\xi}^{k}\right)\right|<2^{-n-1}$ for all $i, j, k<3$ and $\xi=\beta$ or $\xi=\alpha_{\tau}$ for some $\tau \in \Omega$.

By the continuity of the $g_{\sigma 1}^{i}$ 's there is a large enough integer $m>\ell$ such that (D21) and (D22) get transferred to:

(D23) $g_{\sigma 1}^{i}\left[x_{\gamma}^{j}\lceil m] \subseteq I^{i j}\right.$ for all $i, j<3$,

(D24) if $s=x_{\xi}^{k}\left\lceil m\right.$ for some $k<3$ and $\xi \in\{\beta\} \cup\left\{\alpha_{\tau}: \tau \in \Omega\right\}$, then for all $i, j<3$,

$$
\sup _{x \in[s]}\left|g_{\sigma 1}^{i}(x)-g_{\sigma 1}^{j}(x)\right|<\frac{1}{2^{n+1}} .
$$

Thus we have finished our splitting procedure at $\sigma$ and defined $\left(g_{\sigma 0}^{0}, g_{\sigma 0}^{1}, g_{\sigma 0}^{2}\right)$ and $\left(g_{\sigma 1}^{0}, g_{\sigma 1}^{1}, g_{\sigma 1}^{2}\right)$ in $K_{0}^{3}$ at the expense of increasing

(D25) $u_{\rho \varepsilon}^{k}$ to $x_{\alpha_{\rho \varepsilon}}^{k}\lceil m(\rho<\sigma, \varepsilon<2, k<3)$,

(D26) $v_{\rho}^{k}$ to $x_{\alpha_{\rho}}^{k}\lceil m(\rho>\sigma, k<3)$,

which together with

(D27) $u_{\sigma 0}^{k}=x_{\beta}^{k}\lceil m(k<3)$,

(D28) $u_{\sigma 1}^{k}=x_{\gamma}^{k}\lceil m(k<3)$

satisfy the requirements of (D11) and (D12).

This completes the recursive procedure of finding the sequences $\left(g_{\sigma}^{0}, g_{\sigma}^{1}, g_{\sigma}^{2}\right)(\sigma \in$ $\left.2^{<\mathbb{N}}\right)$ and $\left(t_{\sigma}^{0}, t_{\sigma}^{1}, t_{\sigma}^{2}\right)\left(\sigma \in 2^{<\mathbb{N}}\right)$ satisfying the conditions under (D1)-(D10). Having 
done this, for each $a \in 2^{\mathbb{N}}$ and $i<3$ let $x_{a}^{i}$ be the uniquely determined element of the Baire space $\mathbb{N}^{\mathbb{N}}$ which extends $t_{a \uparrow n}^{i}$ for all $n$. Note that $a \mapsto x_{a}^{i}$ is a homeomorphism between the Cantor set $2^{\mathbb{N}}$ and the subset $\left\{x_{a}^{i}: a \in 2^{\mathbb{N}}\right\}$ of the Baire space. Since for each $i<3$ the sequence

$$
g_{s}^{i} \quad\left(s \in 2^{<\mathbb{N}}\right)
$$

is a relatively compact subset of $B_{1}\left(\mathbb{N}^{\mathbb{N}}\right)$ (and in fact included in $K$ ), applying Theorem 11 successively three times gives us a perfect set $P \subseteq 2^{\mathbb{N}}$ and an infinite strictly increasing sequence $\left\{n_{k}\right\}$ of integers such that

$$
\left\{g_{a \uparrow n_{k}}^{i}\right\} \text { is convergent for all } i<3 \text { and } a \in P .
$$

For $i<3$ and $a \in P$, set

$$
g_{a}^{i}=\lim _{k \rightarrow \infty} g_{a \uparrow n_{k}}^{i}
$$

Then $g_{a}^{i}(i<3, a \in P)$ is a sequence of elements of $K$ such that (see (D9) and (D10)):

(D31) $g_{a}^{i}\left(x_{a}^{j}\right) \in \overline{I^{i j}}$ for $a \in P$ and $i, j<3$,

(D32) $g_{a}^{i}\left(x_{b}^{k}\right)=g_{a}^{j}\left(x_{b}^{k}\right)$ for $a \neq b$ in $P$ and $i, j, k<3$.

Choose open rational intervals $J^{i j}(i, j<3)$ such that (see (D5)):

(D33) $\overline{I^{i j}} \subseteq J^{i j}$ for $i, j<3$,

(D34) $J^{00} \cap J^{10}=J^{11} \cap J^{21}=J^{02} \cap J^{22}=\emptyset$.

For $i, j<3$ we define two symmetric relations $A_{0}^{i j}$ and $A_{1}^{i j}$ on $P$ as follows:

(D35) $(x, y) \in A_{0}^{i j}$ iff $x \neq y$ and $g_{a}^{i}\left(x_{b}^{j}\right) \in J^{i j}$ where $a=\min \{x, y\}$ and $b=$ $\max \{x, y\}$

(D36) $(x, y) \in A_{1}^{i j}$ iff $x \neq y$ and $g_{a}^{i}\left(x_{b}^{j}\right) \in J^{i j}$ where $a=\max \{x, y\}$ and $b=$ $\min \{x, y\}$.

(The minimum and maximum here are taken according to the lexicographical ordering of $2^{\mathbb{N}}$.) Note that all these sets $A_{\varepsilon}^{i j}$ are Borel, and this is one of the crucial points of all the construction performed above. Applying Theorem 13 successively twelve times we get a perfect set $R \subseteq P$ such that for all $i, j<3$ and $\varepsilon<2$,

$$
R^{[2]} \cap A_{\varepsilon}^{i j}=\emptyset \quad \text { or } \quad R^{[2]} \subseteq A_{\varepsilon}^{i j} .
$$

For $a \in R$ and $i<3$, define

$$
U\left(g_{a}^{i}\right)=\left\{f \in K: f\left(x_{a}^{j}\right) \in J^{i j} \quad \text { for all } \quad j<3\right\} .
$$

Note that by (D31) and (D33), the set $U\left(g_{a}^{i}\right)$ is an open neighborhood of $g_{a}^{i}$ in $K$. Note also that (D34) leads to

$$
U\left(g_{a}^{i}\right) \cap U\left(g_{a}^{j}\right)=\emptyset \quad \text { for } \quad a \in R \quad \text { and } \quad i \neq j<3 .
$$

On the other hand, the property (D32) leads to the following property of every $k<3$ :

(D40) if $b \neq a$, then either $g_{a}^{i} \in U\left(g_{b}^{k}\right)$ for all $i<3$, or else $g_{a}^{i} \notin U\left(g_{b}^{k}\right)$ for all $i<3$. 
This together with the definition (D35) and (D36) of the set $A_{\varepsilon}^{i j}$ and the property (D37) of our perfect set $R$ gives us the following for every $b \in R$ and $k<3$ :

(D41) $\left\{g_{a}^{i}: i<3, a \in R, a<b\right\} \quad$ is either included in or disjoint from $U\left(g_{b}^{k}\right)$,

(D42) $\left\{g_{a}^{i}: i<3, a \in R, a>b\right\} \quad$ is either included in or disjoint from $U\left(g_{b}^{k}\right)$.

Moreover, we know that these alternatives are uniform on $b \in R$ and $k<3$. Since for a given $b \in R$ the three sets $U\left(g_{b}^{0}\right), U\left(g_{b}^{1}\right)$ and $U\left(g_{b}^{2}\right)$ are pairwise disjoint (see (D39)), there must be $k$ (uniform in $b$ ) such that

$$
U\left(g_{b}^{k}\right) \cap\left\{g_{a}^{i}: i<3, a \in R, a \neq b\right\}=\emptyset .
$$

It follows that $g_{b}^{k}(b \in R)$ is a discrete subspace of $K$ of size continuum. This completes the proof of Theorem 5 and also the proof of Theorem 3.

\section{E. INSERTING THE SPLit INTERVAL INTO $K$}

In this section we give proofs of Theorems 4 and 6 . Note that by Theorems 1 and 3 , it suffices to prove Theorem 4 for separable $K$ which are at most two-to-one preimages of compact metric spaces, and so it suffices to prove only Theorem 6 . Thus, we start with a separable compact set $K$ of the first Baire class on $\mathbb{N}^{\mathbb{N}}$ such that for some countable $D_{0} \subseteq \mathbb{N}^{\mathbb{N}}$, the projection

$$
\pi_{D_{0}}: K \longrightarrow M=\left\{f \uparrow D_{0}: f \in K\right\}
$$

is at most two-to-one. (It is easy to see that the existence of such a $D_{0}$ is indeed equivalent to the assumption that $K$ is at most a two-to-one preimage of a compact metric space.) Let $K_{0}$ be a fixed countable dense subset of $K$. As before, we may assume that every $f \in K_{0}$ is a continuous function on $\mathbb{N}^{\mathbb{N}}$. Clearly, we may assume that $K$ itself is not metrizable which amounts to the fact that there is no countable set $D \subseteq \mathbb{N}^{\mathbb{N}}$ for which the corresponding projection $\pi_{D}$ is one-to-one. This means that by recursion on countable ordinals $\alpha$, we can choose elements $f_{\alpha}^{0}$ and $f_{\alpha}^{1}$ of $K$ and an element $x_{\alpha}$ of the Baire space such that the following conditions are satisfied:

(E1) $f_{\alpha}^{0} \uparrow D_{0}=f_{\alpha}^{1} \uparrow D_{0}$,

(E2) $f_{\alpha}^{0}\left(x_{\alpha}\right) \neq f_{\alpha}^{1}\left(x_{\alpha}\right)$,

(E3) $f_{\beta}^{0}\left(x_{\alpha}\right)=f_{\beta}^{1}\left(x_{\alpha}\right)$ whenever $\quad \alpha<\beta$,

(E4) $f_{\alpha}^{i} \uparrow D_{0} \neq f_{\beta}^{j}\left\lceil D_{0}\right.$ whenever $\quad \alpha \neq \beta$ and $i, j<2$.

(To get (E4) we use the fact that $K$ is not metrizable and the fact that the projection $\pi_{D_{0}}$ is an at most two-to-one mapping.) Going to a subsequence, we may (and will) assume to have two rational intervals $I^{0}$ and $I^{1}$ with disjoint closures such that:

$$
f_{\alpha}^{i}\left(x_{\alpha}\right) \in I^{i} \text { for all } \alpha \text { and } i .
$$

For every positive integer $n$ we fix an enumeration $B_{n k}(k \in \mathbb{N})$ of all open rational intervals of length $\leq 2^{-n}$. Let $d_{n}(n \in \mathbb{N})$ be an enumeration of $D_{0}$ with infinite repetitions. Then every element $t$ of $\mathbb{N}<\mathbb{N}$ determines the basic open set

$$
B(t)=\left\{h \in \mathbb{R}^{D_{0}}: h\left(d_{n}\right) \in B_{n t(n)} \quad \text { for every } n \in|t|\right\}
$$

of $\mathbb{R}^{D_{0}}$. Note that $B(t)\left(t \in \mathbb{N}^{<N}\right)$ is a basis of the cube $\mathbb{R}^{D_{0}}$. Note moreover that the basis gives us a natural way to associate perfect subsets of $\mathbb{R}^{D_{0}}$ to perfect subtrees of $\mathbb{N}<\mathbb{N}$, but we shall need a slightly stronger notion of splitting between 
nodes of $\mathbb{N}<\mathbb{N}$ that would make this correspondence even more precise. Thus, we say that two nodes $s$ and $t$ of $\mathbb{N}<\mathbb{N}$ properly split if there is an $n$ in their common domain such that not only $s(n) \neq t(n)$ but the rational intervals $B_{n s(n)}$ and $B_{n t(n)}$ have disjoint closures. For a given countable ordinal $\alpha$ let $h_{\alpha} \in \mathbb{R}^{D_{0}}$ denote the common projection $f_{\alpha}^{0} \uparrow D_{0}=f_{\alpha}^{1} \uparrow D_{0}$. Let $T$ be the set of all pairs $\left(t^{0}, t^{1}\right)$ of elements of $\mathbb{N}<\mathbb{N}$ of the same length such that

there exist uncountably many $\alpha$ 's such that

$$
x_{\alpha} \text { extends } t^{0} \text { and } h_{\alpha} \text { belongs to } B\left(t^{1}\right) .
$$

It is easily seen that the tree $T$ is perfect in the following sense:

(E7) for every $\left(s^{0}, s^{1}\right) \in T$ there exist two extensions $\left(t^{0}, t^{1}\right)$ and $\left(u^{0}, u^{1}\right)$ on the same level of $T$ such that $t^{0} \neq u^{0}$ and such that $t^{1}$ and $u^{1}$ properly split.

Using these objects it should be clear that the recursive construction of $\S \mathrm{D}$ adapts, giving us two sequences

$$
\begin{gathered}
\left(t_{\sigma}^{0}, t_{\sigma}^{1}\right) \in T \quad\left(\sigma \in 2^{<\mathbb{N}}\right), \\
\left(g_{\sigma}^{0}, g_{\sigma}^{1}\right) \in K_{0}^{2} \quad\left(\sigma \in 2^{<\mathbb{N}}\right),
\end{gathered}
$$

such that the following five conditions are satisfied:

(E8) if $\tau$ extends $\sigma$, then $\left(t_{\tau}^{0}, t_{\tau}^{1}\right)$ extends $\left(t_{\sigma}^{0}, t_{\sigma}^{1}\right)$,

(E9) $\left(t_{\sigma 0}^{0}, t_{\sigma 0}^{1}\right)$ and $\left(t_{\sigma 1}^{0}, t_{\sigma 1}^{1}\right)$ are two extensions of $\left(t_{\sigma}^{0}, t_{\sigma}^{1}\right)$ to the same level of $T$ such that $t_{\sigma 0}^{0} \neq t_{\sigma 1}^{0}$ and such that $t_{\sigma 0}^{1}$ and $t_{\sigma 1}^{1}$ properly split,

(E10) $g_{\sigma}^{i}\left[t_{\sigma}^{0}\right] \subseteq I^{i}$ for all $\sigma \in 2^{<\mathbb{N}}$ and $i<2$,

(E11) $g_{\sigma}^{i}\left\lceil D_{0} \in B\left(t_{\sigma}^{1}\right)\right.$ for all $\sigma \in 2^{<\mathbb{N}}$ and $i<2$,

(E12) if $\sigma \neq \tau$ have the same length $n$, then $\sup _{x \in\left[t_{\sigma}^{0}\right]}\left|g_{\tau}^{0}(x)-g_{\tau}^{1}(x)\right| \leq 1 / 2^{n}$.

For $a \in 2^{\mathbb{N}}$, let $x_{a}$ be the element of the Baire space determined by the sequence $\left\{t_{x \nmid n}^{0}\right\}$. Then $a \mapsto x_{a}$ is a homeomorphism between the Cantor cube and the perfect subset $\left\{x_{a}: a \in 2^{\mathbb{N}}\right\}$ of the Baire space. Note that for a fixed $a \in 2^{\mathbb{N}}$, the sequence

$$
B\left(t_{x\lceil n}^{1}\right) \quad(n \in \mathbb{N})
$$

of basic open sets determines a unique element $h_{a}$ of the cube $\mathbb{R}^{D_{0}}$. Note moreover that $a \mapsto h_{a}$ is a homeomorphism between the Cantor cube and the perfect set

$$
\left\{h_{a}: a \in 2^{\mathbb{N}}\right\} \subseteq \mathbb{R}^{D_{0}} .
$$

For $i<2$ the sequence $g_{\sigma}^{i}\left(\sigma \in 2^{<\mathbb{N}}\right)$ is relatively compact in $B_{1}\left(\mathbb{N}^{\mathbb{N}}\right)$ as it is included in $K$, so a successive application of Theorem 11 gives us a perfect set $P \subseteq 2^{\mathbb{N}}$ and an infinite strictly increasing sequence $\left\{n_{k}\right\}$ of integers such that

$$
\left\{g_{a \uparrow n_{k}}^{i}\right\} \text { is pointwise convergent for every } a \in P \text { and } i<2 \text {. }
$$

So, for $i<2$ and $a \in P$ we may let

$$
g_{a}^{i}=\lim _{k \rightarrow \infty} g_{a \nmid n_{k}}^{i} .
$$

Then $g_{a}^{i}(i<2, a \in P)$ is a sequence of elements of $K$ such that (see (E10), (E11) and $(\mathrm{E} 12))$

(E15) $g_{a}^{i}\left(x_{a}\right) \in \overline{I^{i}}$ for $i<2$ and $a \in P$,

(E16) $g_{a}^{0} \uparrow D_{0}=h_{a}=g_{a}^{1} \uparrow D_{0} \quad$ for $\quad a \in P$,

(E17) $g_{a}^{0}\left(x_{b}\right)=g_{a}^{1}\left(x_{b}\right)$ for $a \neq b$ in $P$. 
Note that since $\overline{I^{0}}$ and $\overline{I^{1}}$ are disjoint, the functions $g_{a}^{0}$ and $g_{a}^{1}$ are different. Since $\pi_{D_{0}}$ is at most two-to-one, we conclude that for each $a \in P$,

$$
\pi_{D_{0}}^{-1}\left(h_{a}\right)=\left\{g_{a}^{0}, g_{a}^{1}\right\} .
$$

Choose two open rational intervals $J^{0}$ and $J^{1}$ such that

(E19) $\overline{I^{i}} \subseteq J^{i}$ for $i<2$, and

(E20) $J^{0} \cap J^{1}=\emptyset$.

For $i, j<2$ we define two symmetric subsets $A_{0}^{i j}$ and $A_{1}^{i j}$ of $P^{2}$ as follows:

(E21) $(x, y) \in A_{0}^{i j}$ iff $x \neq y$ and $g_{a}^{i}\left(x_{b}\right) \in J^{j}$ where $a=\min \{x, y\}$ and $b=$ $\max \{x, y\}$

(E22) $(x, y) \in A_{1}^{i j}$ iff $x \neq y$ and $g_{a}^{i}\left(x_{b}\right) \in J^{j}$ where $a=\max \{x, y\}$ and $b=$ $\min \{x, y\}$.

(Here, again, the minimum and the maximum are taken with respect to the lexicographical ordering of $2^{\mathbb{N}}$.) One of the crucial points of the whole construction is the fact that these sets $A_{\varepsilon}^{i j}$ are all Borel subsets of $P^{2}$. Applying Theorem 13 eight times (actually, four times since clearly $A_{\varepsilon}^{0 j}=A_{\varepsilon}^{1 j}$ by (E17)), we get a perfect set $R \subseteq P$ such that for all $i, j<2$ and $\varepsilon<2$,

$$
R^{[2]} \subseteq A_{\varepsilon}^{i j} \quad \text { or } \quad R^{[2]} \cap A_{\varepsilon}^{i j}=\emptyset .
$$

For $a \in R$ and $i<2$, set

$$
U\left(g_{a}^{i}\right)=\left\{f \in K: f\left(x_{a}\right) \in J^{i}\right\} .
$$

Then by (E15) and (E19), the set $U\left(g_{a}^{i}\right)$ is an open neighborhood of $g_{a}^{i}$ in $K$. By (E20) and (E17) we moreover have the following properties:

(E24) $U\left(g_{a}^{0}\right) \cap U\left(g_{a}^{1}\right)=\emptyset$,

(E25) for $a \neq b$ and $i<2$, the neighborhood $U\left(g_{b}^{i}\right)$ contains either both or none of the points $g_{a}^{0}$ and $g_{a}^{1}$.

Combining this with (E23) we get the following two properties uniformly on $b \in R$ and $i<2$ :

(E26) the set $U\left(g_{b}^{i}\right)$ either contains or is disjoint from $\left\{g_{a}^{j}: j<2, a \in R, a<b\right\}$, (E27) the set $U\left(g_{b}^{i}\right)$ either contains or is disjoint from $\left\{g_{a}^{j}: j<2, a \in R, a>b\right\}$.

Case 1. For some $i<2$ and all $b \in R$, the set $U\left(g_{b}^{i}\right)$ is disjoint from

$$
\left\{g_{a}^{j}: j<2, a \in R, a \neq b\right\} .
$$

Since $a \mapsto h_{a}$ is a homeomorphism and since (E18) holds, if a sequence $\left\{a_{n}\right\}$ of elements of $R$ converges to some $b \in R$, the corresponding sequences $\left\{g_{a_{n}}^{j}\right\}$ must accumulate to some element of the pair $\left\{g_{b}^{0}, g_{b}^{1}\right\}$. By our assumption the neighborhood $U\left(g_{b}^{i}\right)$ separates $g_{b}^{i}$ from everything else, and so all those sequences must accumulate to the other point $g_{b}^{1-i}$ of the pair. Since this is uniform in $b$ and $i$ we conclude that the space

$$
\left\{g_{b}^{k}: k<2, b \in R\right\}
$$

is homeomorphic to the duplicate of the perfect set $\left\{h_{b}: b \in R\right\}$. This gives us the alternative (b) of Theorem 6. 
Case 2. For all $i<2$ and $b \in R$, the set $U\left(g_{b}^{i}\right)$ intersects $\left\{g_{a}^{j}: j<2, a \in R, a \neq\right.$ $b\}$. Since $U\left(g_{b}^{0}\right)$ and $U\left(g_{b}^{1}\right)$ are disjoint, we have uniformly on $b \in R$ (see (E26) and (E27)):

$$
\begin{aligned}
& \left\{g_{a}^{j}: j<2, a \in R, a<b\right\} \subseteq U\left(g_{b}^{0}\right) \\
& \quad \text { and }\left\{g_{a}^{j}: j<2, a \in R, a>b\right\} \subseteq U\left(g_{b}^{1}\right),
\end{aligned}
$$

or vice versa. For concreteness we assume that the first alternative happens for all $b \in R$. Note that this means that the subspace

$$
\left\{g_{b}^{k}: k<2, b \in R\right\}
$$

of $K$ is homeomorphic to the lexicographical product $R \times 2$ via the mapping $(b, k) \mapsto$ $g_{b}^{k}$. It follows that our compactum $K$ contains a copy of the split $2^{\mathbb{N}} \times 2$ of the Cantor set $2^{\mathbb{N}}$. Note that $2^{\mathbb{N}} \times 2$ has only countably many isolated points. If we remove them, then a standard back-and-forth argument shows that the so-obtained subspace of $2^{\mathbb{N}} \times 2$ is homeomorphic (and order-isomorphic) to the split interval. This completes the proof of Theorem 6 as well as the proof of Theorem 4 .

\section{F. Inserting the Alexandroff Duplicate into $K$}

In this section we give a proof of Theorem 7 which reveals the critical role the Alexandroff duplicate $D\left(2^{\mathbb{N}}\right)$ plays inside the class of premetric compacta of degree 2 and of the first Baire class. This result together with Theorem 6 proved in $\S \mathrm{E}$ shows that the nonmetric compacta of this class are naturally divided into two classes with critical members $S\left(2^{\mathbb{N}}\right)$ and $D\left(2^{\mathbb{N}}\right)$, respectively. Most of the ideas of the proof have already been exposed in the previous sections, so we decided to make the proof rather brief relying on the reader's understanding of the previous material.

Let $K$ be a given separable premetric compactum of degree 2 and of first Baire class. It is clear that the conditions (a) and (b) both imply (c) so we are left to show that if one of (a) and (b) fails for $K$, then $K$ contains a copy of $D\left(2^{\mathbb{N}}\right)$. Note that by Theorem 1 , if (a) fails, then $K$ contains an uncountable discrete subspace and therefore (b) also fails. Thus we are left to show that if (b) fails, then $D\left(2^{\mathbb{N}}\right)$ embeds into $K$. If (b) fails, then a simple recursion on countable ordinals $\alpha$ gives us a sequence $f_{\alpha}$ of elements of $K$ and a sequence $U_{\alpha}$ of their open neighborhoods such that

$$
f_{\beta} \notin U_{\alpha} \text { whenever } \alpha<\beta \text {. }
$$

Keeping the notation from the previous section, note that if $h_{\alpha}=f_{\alpha} \uparrow D_{0}$, then for all but countably many $\alpha$ 's the preimage $\pi_{D_{0}}^{-1}\left(h_{\alpha}\right)$ has two elements, say $g_{\alpha}^{0}$ and $g_{\alpha}^{1}$. Pick $x_{\alpha} \in \mathbb{N}^{\mathbb{N}}$ such that $g_{\alpha}^{0}\left(x_{\alpha}\right) \neq g_{\alpha}^{1}\left(x_{\alpha}\right)$. Going to a subsequence, we assume that we have two rationals $\delta<\varepsilon$ such that

$$
g_{\alpha}^{0}\left(x_{\alpha}\right)<\delta<\varepsilon<g_{\alpha}^{1}\left(x_{\alpha}\right) \text { for all } \alpha .
$$

For concreteness, assume also that $f_{\alpha}=g_{\alpha}^{0}$ for all $\alpha$. Since a neighborhood basis of $g_{\alpha}^{0}$ can be obtained by intersecting any open set $U$ which separates it from $g_{\alpha}^{1}$ with the $\pi_{D_{0}}$-preimage of a basic open set of $\mathbb{R}^{D_{0}}$ containing $h_{\alpha}$, we conclude that for every countable set $D \supseteq D_{0}$, for all but countably many $\alpha$ 's the restrictions $g_{\alpha}^{0}\lceil D$ and $g_{\alpha}^{1}\lceil D$ must be equal. So, going to a subsequence we may assume that

$$
g_{\beta}^{0}\left(x_{\alpha}\right)=g_{\beta}^{1}\left(x_{\alpha}\right) \quad \text { whenever } \quad \alpha<\beta \text {. }
$$


For the same reasons we may assume that for some fixed basic open set $B$ of the cube $\mathbb{R}^{D_{0}}$,

$$
U_{\alpha}=\pi_{D_{0}}^{-1}(B) \cap\left\{f \in K: f\left(x_{\alpha}\right)<\varepsilon\right\} \text { for all } \alpha .
$$

Combining this with the property (F1) of the sequence $U_{\alpha}$ of separating neighborhoods, we conclude that

$$
g_{\beta}^{0}\left(x_{\alpha}\right) \geq \varepsilon \quad \text { whenever } \quad \alpha<\beta .
$$

Hence we have here the situation analogous to that of (E1)-(E5) of $\S \mathrm{E}$. So we can rely on the construction of $\S \mathrm{E}$ and assume that we have a perfect set $P \subseteq 2^{\mathbb{N}}$ and for each $a \in P$, a pair $\left\{g_{a}^{0}, g_{a}^{1}\right\}$ of elements of $K$, a point $x_{a} \in \mathbb{N}^{\mathbb{N}}$, and a point $h_{a} \in \mathbb{R}^{D_{0}}$ such that

(F6) $a \mapsto x_{a}$ and $a \mapsto h_{a}$ are continuous and one-to-one,

(F7) $g_{a}^{0} \uparrow D_{0}=g_{a}^{1} \uparrow D_{0}=h_{a}$,

(F8) $g_{a}^{0}\left(x_{a}\right) \leq \delta<\varepsilon \leq g_{a}^{1}\left(x_{\alpha}\right)$,

(F9) $g_{b}^{0}\left(x_{a}\right)=g_{b}^{1}\left(x_{a}\right) \geq \varepsilon$ whenever $a \neq b$.

Let

$$
H=\left\{g_{b}^{0}, g_{b}^{1}: b \in P\right\}
$$

For $a \in P$, set

$$
U\left(g_{a}^{0}\right)=\left\{f \in K: f\left(x_{a}\right)<\varepsilon\right\} .
$$

Then $U\left(g_{a}^{0}\right)$ is an open neighborhood of $g_{a}^{0}$ which separates it from any other point of $H$. On the other hand, note that if $\left\{a_{n}\right\}$ is a sequence of elements of $P$ which accumulates to some $a \in P$, then both sequences $\left\{g_{a_{n}}^{0}\right\}$ and $\left\{g_{a_{n}}^{1}\right\}$ must accumulate to $g_{a}^{1}$. This means that $H$ behaves topologically as the Alexandroff duplicate of the perfect set $\left\{h_{a}: a \in P\right\}$. This finishes the proof.

\section{G. Points in separable compact subsets of the first Baire Class}

The purpose of this section is to give a proof of Theorem 9. We shall need a formulation of the Bourgain-Fremlin-Talagrand Theorem due to R. Pol [Po3]. It is interesting that similar ideas appear also in a paper of G. Debs [D] which is concerned with finding an effective version of the Bourgain-Fremlin-Talagrand Theorem. For the rest of this section $K$ will be a fixed compact subset of the first Baire class on some Polish space $X$ and $\left\{f_{k}\right\}$ is a fixed enumeration of some countable dense subset of $K$. Increasing the topology of $X$ but without changing its Polishness we may (and will) assume that each $f_{k}$ is actually a continuous function on $X$. In fact, it will be clear from the proof that Theorem 9 is true for any compact $K \subseteq \mathcal{B}_{1}(X)$ with the property that $K \cap \mathcal{C}(X)$ is dense in $K$. Since the proof of the following lemma is given in [Po3] only in a special case, for completeness, we sketch the argument.

Lemma 6. For every ultrafilter $\mathcal{U}$ on $K$ that converges to some point $z \in K$ there is a sequence $\left\{B_{n}\right\}$ of members of $\mathcal{U}$ that converges to $z$. 
Proof. For a given pair of rationals $p<q$ we define a derivation operation for subsets of $X$ as follows:

$$
\begin{aligned}
\partial F=\left\{x \in F:\left\{g \in K: \inf _{a \in V \cap F} g(a)<p<q<\sup _{a \in V \cap F} g(a)\right\} \in \mathcal{U}\right. & \\
& \text { for every open } V \ni x\} .
\end{aligned}
$$

One extends this to the transfinite sequence of derivatives in the usual way:

$$
\begin{aligned}
\partial_{0} F & =F, \\
\partial_{\alpha+1} F & =\partial\left(\partial_{\alpha} F\right), \\
\partial_{\lambda} F & =\bigcap_{\alpha<\lambda} \partial_{\alpha} F \quad(\lambda \text { limit }>0) .
\end{aligned}
$$

The lemma is proved once we show that the transfinite sequence of derivatives of $X$ vanishes. Otherwise, there will be a closed nonempty subset $F$ of $X$ such that $\partial F=F$. By recursion on $s \in 2^{<\mathbb{N}}$ we build a sequence $U_{s}\left(s \in 2^{<\mathbb{N}}\right)$ of nonempty relatively open subsets of $F$ and an increasing sequence $\left\{i_{n}\right\}$ of integers such that

(G1) $\operatorname{diam}\left(U_{s}\right) \leq 2^{-|s|}$,

(G2) $\overline{U_{s} \sim 0} \subseteq U_{s} \cap\left\{f_{i_{|s|}}<p\right\}$,

(G3) $\overline{U_{s\urcorner 1}} \subseteq U_{s} \cap\left\{f_{i_{|s|}}>q\right\}$.

(Here diameter is defined relative to a fixed complete metric on $X$ that defines its topology.) Suppose $U_{s}(|s| \leq n)$ and $i_{k}(k \leq n)$ have been defined. From the assumption $\partial F=F$ we infer that

$$
K_{s}=\left\{g \in K: \inf _{a \in U_{s}} g(a)<p<q<\sup _{a \in U_{s}} g(a)\right\}
$$

belongs to $\mathcal{U}$ for all $s$ of length $\leq n$. So we can fix a $g$ in $\bigcap_{|s| \leq n} K_{s}$. For each $s$ of length $\leq n$ fix points $a_{s \frown 0}$ and $a_{s \frown 1}$ in $U_{s} \cap F$ such that $g\left(a_{s \frown 0}\right)<p$ and $g\left(a_{s \frown 1}\right)>q$. Since $\left\{f_{i}\right\}$ is dense in $K$ there is an index $i_{n+1}>i_{n}$ such that $f_{i_{n+1}}\left(a_{s \sim 0}\right)<p$ and $f_{i_{n+1}}\left(a_{s\urcorner 1}\right)>q$ for all $s$ of length $\leq n$. Since $f_{i_{n+1}}$ is a continuous function, it is possible to pick relatively open subsets $U_{s \frown 0}$ and $U_{s \frown 1}$ of $F$ satisfying the conditions (G1)-(G3).

For $a \in 2^{\mathbb{N}}$ let $x_{a}$ be the unique point of $F$ belonging to $U_{a \uparrow n}$ for all $n$. The mapping $n \mapsto f_{i_{n}}$ from $\mathbb{N}$ into $K$ naturally extends to a continuous mapping $\xi \mapsto f_{\xi}$ from $\beta \mathbb{N}$ into $K$ given by

$$
g_{\xi}(x)=\lim _{n \rightarrow \xi} g_{i_{n}}(x) .
$$

We shall reach the desired contradiction once we show (cf. the proof of Theorem 11) that $g_{\xi} \neq g_{\eta}$ whenever $\xi \neq \eta$. Choose $a \in 2^{\mathbb{N}}$ such that

$$
\{n: a(n)=0\} \in \xi \quad \text { and } \quad\{n: a(n)=1\} \in \eta .
$$

Then by the construction of $\left\{U_{s}\right\}$ and $\left\{i_{n}\right\}$ we have that $g_{\xi}\left(x_{a}\right) \leq p$ while $g_{\eta}\left(x_{a}\right) \geq q$ and this shows that $g_{\xi}$ and $g_{\eta}$ are different, finishing the proof of Lemma 6 .

Remark 3. The conclusion of Lemma 6 is of course much stronger than the Fréchet property of $K$, the way Bourgain, Fremlin and Talagrand state their result. However, there is an important difference between the two results. While the separability of $K$ (or more generally the assumption that $K \cap \mathcal{C}(K)$ is dense in $K$ ) is a crucial assumption for the conclusion of Lemma 6 , no such assumption is needed for proving that $K$ is only a Fréchet space. On the other hand, Lemma 6 does 
immediately give us the full Bourgain-Fremlin-Talagrand theorem. For this one only needs to recall a lemma of Rosenthal [R3, Lemma 3.9] which says that every compact subset of the first Baire class is countably tight reducing thus the Fréchet property of an arbitrary $K$ to the Fréchet property of separable $K$.

For the rest of this section we fix a non- $G_{\delta}$-point of $K$ and work towards the conclusion of Theorem 9. Clearly we may assume that the chosen point is the constantly-zero function $\overrightarrow{0}$. We also assume that $f_{n} \neq \overrightarrow{0}$ for all $n$. It is now quite natural to consider the following ideal of subsets of $\mathbb{N}$ :

$$
\mathcal{I}=\left\{a: \overrightarrow{0} \text { is not in the closure of }\left\{f_{n}\right\}_{n \in a}\right\} .
$$

Thus, our assumption that $\overrightarrow{0}$ is not a $G_{\delta}$ point of $K$ reduces to the assumption that $\mathcal{I}$ is not a countably generated ideal. It is also natural to consider the following ideal of subsets of $\mathbb{N}$, the orthogonal of $\mathcal{I}$ :

$$
\mathcal{I}^{\perp}=\left\{b:\left\{f_{n}\right\}_{n \in b} \text { converges to } \overrightarrow{0}\right\} .
$$

The orthogonal $\mathcal{I}^{\perp}$ also captures many essential properties of the point $\overrightarrow{0}$ inside the space $K$. For example, the Bourgain-Fremlin-Talagrand Theorem reduces to the fact that every $c \notin \mathcal{I}$ contains an infinite subset which belongs to $\mathcal{I}^{\perp}$. We shall also need the following lemma which is closely related to an old result of Szlenk [Sz] which shows that a point of a compact subset of the first Baire class has the diagonal-sequence property only in the extreme case when it has a countable neighborhood base.

Lemma 7. The following conditions are equivalent:

(1) $\overrightarrow{0}$ is a $G_{\delta}$ point of $K$.

(2) If for each integer $k$ we have a sequence $\left\{x_{n}^{k}\right\}$ of elements of $K$ that converges to $\overrightarrow{0}$, then there is a diagonal sequence $\left\{x_{n(k)}^{k}\right\}$ that converges to $\overrightarrow{0}$.

Proof. It is clear that (1) implies (2). So let us assume (1) is false and work towards showing the diagonal-sequence property (2) must also be false. Note that $\mathcal{I}$ is an analytic subset of the power set of $\mathbb{N}$. So Theorem 3 of [To3] applies to the pre-gap formed by $\mathcal{I}$ and $\mathcal{I}^{\perp}$. Note that the Bourgain-Fremlin-Talagrand Theorem gives us the equation

$$
\mathcal{I}^{\perp \perp}=\mathcal{I} .
$$

From this equation and our assumption that (1) is false (or equivalently, that $\mathcal{I}$ is not countably generated) we see that the second alternative of Theorem 3 of [To3] must hold. In other words there must be a collection $\Sigma$ of finite sets of integers such that

(G4) $\emptyset \in \Sigma$,

(G5) $b_{\sigma}=\{i: i>\max (\sigma)$ and $\sigma \cup\{i\} \in \Sigma\}$ belongs to $\mathcal{I}^{\perp}$ for every $\sigma \in \Sigma$,

(G6) every subset $a$ of $\mathbb{N}$ with the property that $a \cap\{0, \ldots, n\} \in \Sigma$ for all $n \in \mathbb{N}$ must belong to $\mathcal{I}$.

Suppose $c \cap b_{\sigma} \neq \emptyset$ for all $\sigma \in \Sigma$. Define an increasing sequence $\left\{i_{n}\right\}$ of integers recursively as follows:

$$
i_{n}=\min \left(b_{\left\{i_{k}: k<n\right\}} \cap c\right) .
$$


Then $a=\left\{i_{n}\right\}$ is an infinite subset of $c$ which satisfies the hypothesis of (G6) and therefore it must belong to $\mathcal{I}$. It follows that $c$ is not a member of $\mathcal{I}^{\perp}$. This finishes the proof.

We shall, however, need the following property of $\mathcal{I}$, a proof of which involves essentially also its orthogonal $\mathcal{I}^{\perp}$. Recall that $\mathbb{N}^{\mathbb{N}}$ can also be considered as a lattice under the ordering $x \leq y$ meaning that $x(n) \leq y(n)$ for all $n$. We also consider $\mathcal{I}$ as a lattice under the inclusion ordering. A cofinal subset of $\mathbb{N}^{\mathbb{N}}$ is simply a subset $D$ with the property that for every $x \in \mathbb{N}^{\mathbb{N}}$, there is $y \in D$ such that $x \leq y$.

Lemma 8. If $\overrightarrow{0}$ is not a $G_{\delta}$ point in $K$, then there is a monotonic map $\Phi: \mathcal{I} \rightarrow \mathbb{N}^{\mathbb{N}}$ whose range is cofinal in $\mathbb{N}^{\mathbb{N}}$.

Proof. By Lemma 7 there is a sequence $\left\{b_{i}\right\}$ of elements of $\mathcal{I}^{\perp}$ with the property that no member of $\mathcal{I}^{\perp}$ intersects each $b_{i}$. Define $\Phi: \mathcal{I} \rightarrow \mathbb{N}^{\mathbb{N}}$ as follows:

$$
\Phi(a)(n)=\min \left\{m \in \mathbb{N}: a \cap b_{i} \subseteq\{0, \ldots, m\} \text { for all } i \leq n\right\} .
$$

For $k \in \mathbb{N}$ define $\Phi_{k}: \mathcal{I} \rightarrow \mathbb{N}^{\mathbb{N}}$, the $k$-translation of $\Phi$, as follows:

$$
\Phi_{k}(a)(n)=\Phi(a)(n+k) .
$$

Then it is possible to show (see the proof of Theorem 5 of [To3]) that there must be a $k$ such that the range of $\Phi_{k}$ is cofinal in $\mathbb{N}^{\mathbb{N}}$.

Remark 4. It should be noted that Lemma 8 can also be deduced from the main result of Krawczyk [Kr], the paper that contains the first substantial progress towards the problem of Pol [Po1].

We shall also be interested in the following natural subset of $\mathcal{I}$ :

$$
\mathcal{I}^{0}=\left\{b:\left\{f_{n}\right\}_{n \in b} \text { converges to some point } \neq \overrightarrow{0}\right\} .
$$

Note that $\mathcal{I}^{0}$ and $\mathcal{I}^{\perp}$ are both coanalytic subsets of the power-set of $\mathbb{N}$ so we can find two Schoenfield trees $S_{0}$ and $S_{1}$ which project onto $\mathcal{I}^{0}$ and $\mathcal{I}^{\perp}$, respectively (see [Ke]). Thus, members of $S_{0}$ and $S_{1}$ are pairs $(s, t)$, where $s$ is a finite increasing sequence of integers and $t$ is a finite increasing sequence of countable ordinals and moreover $s$ and $t$ are of equal length, i.e., $|s|=|t|$. We shall actually identify $s$ and $t$ with the finite sets of integers or ordinals, respectively, that they enumerate. Then the ordering relation on $S_{0}$ and $S_{1}$ is simply coordinatewise end-extension. Let $\left[S_{0}\right]$ and $\left[S_{1}\right]$ be the sets of all infinite branches of $S_{0}$ and $S_{1}$, respectively. Thus, a member of $\left[S_{0}\right]$ or $\left[S_{1}\right]$ can naturally be identified with a pair of sets $(a, b)$ where $a$ is an infinite set of integers and $b$ is an infinite set of countable ordinals of order-type $\omega$ such that for any $n \in \mathbb{N}$, if $a[n]$ and $b[n]$ are the initial parts of $a$ and $b$ respectively consisting of the first $n$ many of their elements, then $(a[n], b[n])$ belongs to the tree $S_{0}$ or $S_{1}$, respectively. The fact that $\mathcal{I}^{0}$ is a projection of $S_{0}$ and that $\mathcal{I}^{\perp}$ is a projection of $S_{1}$ simply means that an infinite set of integers $a$ belongs to $\mathcal{I}^{0}$ or $\mathcal{I}^{\perp}$ if and only if there is a set $b$ of countable ordinals of order-type $\omega$ such that $(a, b)$ is a branch of $S_{0}$ or $S_{1}$, respectively. In fact, we shall be more interested in the tree formed from $S_{0}$ and $S_{1}$, as follows:

$$
S=\left\{\left(\left(s_{0}, t_{0}\right),\left(s_{1}, t_{1}\right)\right) \in S_{0} \times S_{1}: s_{0} \cap s_{1}=\emptyset \text { and }\left|s_{0}\right|=\left|s_{1}\right|=\left|t_{0}\right|=\left|t_{1}\right|\right\} .
$$

Of course, we take the coordinatewise ordering on $S$. For a subtree $T$ of $S$, which we always implicitly assume to be downwards closed, its derivative $\partial T$, another downwards closed subtree of $S$, is defined as follows: We put $\left(\left(s_{0}, t_{0}\right),\left(s_{1}, t_{1}\right)\right)$ in $\partial T$ 
if we can find two of its proper extensions $\left(\left(s_{0}^{\prime}, t_{0}^{\prime}\right),\left(s_{1}^{\prime}, t_{1}^{\prime}\right)\right)$ and $\left(\left(s_{0}^{\prime \prime}, t_{0}^{\prime \prime}\right),\left(s_{1}^{\prime \prime}, t_{1}^{\prime \prime}\right)\right)$ on the same level of the tree $T$ such that

$$
\left(s_{0}^{\prime} \cap s_{1}^{\prime \prime}\right) \cup\left(s_{0}^{\prime \prime} \cap s_{1}^{\prime}\right) \neq \emptyset .
$$

The operation can be extended into the transfinite in a natural way:

$$
\begin{aligned}
\partial_{0} T & =T \\
\partial_{\alpha+1} T & =\partial\left(\partial_{\alpha} T\right), \\
\partial_{\lambda} T & =\bigcap_{\alpha<\lambda} \partial_{\alpha} T \quad(\lambda \text { limit }>0) .
\end{aligned}
$$

We are now in position to state the crucial lemma of the proof of Theorem 9.

Lemma 9. The transfinite sequence of derivatives of the tree $S$ does not vanish.

Proof. Let $\alpha_{0}$ be the minimal ordinal $\alpha$ such that $\partial_{\alpha+1} S=\partial_{\alpha} S$. We have to show that $\partial_{\alpha_{0}} S \neq \emptyset$. For a subtree $T$ of $S$ and $p \in T$, let $T_{p}$ be the subtree of $T$ consisting of all nodes of $T$ comparable to $p$. The set $[T]$ of all infinite branches of $T$ is simply this subset of $\left[S_{0}\right] \times\left[S_{1}\right]$ consisting of pairs of branches whose initial restrictions belong to $T$. We shall be interested in the sets

$$
\begin{aligned}
\operatorname{proj}[T] & =\left\{\left(a_{0}, a_{1}\right):\left(\left(a_{0}, b_{0}\right),\left(a_{1}, b_{1}\right)\right) \in[T] \text { for some } b_{0} \text { and } b_{1}\right\}, \\
\operatorname{proj}_{0}[T] & =\left\{a_{0}:\left(a_{0}, a_{1}\right) \in \operatorname{proj}[T] \text { for some } a_{1}\right\} .
\end{aligned}
$$

For $\alpha<\alpha_{0}$ and $p \in \partial_{\alpha} S \backslash \partial_{\alpha+1} S$, let

$$
c(\alpha, p)=\text { the union of the family } \operatorname{proj}_{0}\left[\left(\partial_{\alpha} S\right)_{p}\right] .
$$

Finally, set

$$
\mathcal{C}=\left\{c(\alpha, p): \alpha<\alpha_{0}, p \in \partial_{\alpha} S \backslash \partial_{\alpha+1} S\right\} .
$$

Note that $\alpha_{0}$ is an ordinal $<\omega_{2}$ so the collection $\mathcal{C}$ does not have more than $\aleph_{1}$ many elements. (Some of the $c(\alpha, p)$ may be empty.) We intend to do some manipulations with $\mathcal{C}$ in the style of Hausdorff and Rothberger, so it will be convenient to assume the following combinatorial property considered first by Rothberger himself (see [Ro], $[\mathrm{F}])$ :

For every filter-base $\mathcal{F}$ of size $\aleph_{1}$ of infinite subsets of $\mathbb{N}$ there is an infinite

subset $c$ of $\mathbb{N}$ such that $c \backslash b$ is finite for all $b \in \mathcal{F}$.

It is well-known (see [F]) that there is always a forcing extension $V^{(\mathcal{B})}$ which satisfies (P) and preserves the initial ordinals $\omega_{1}$ and $\omega_{2}$. Considering, without loss of generality, the case $K \subseteq \mathcal{B}_{1}\left(\mathbb{N}^{\mathbb{N}}\right)$ and referring to $\S \mathrm{A}$, the extension $V^{(\mathcal{B})}$ has its own versions $\hat{K}, \hat{\mathcal{I}}, \hat{\mathcal{I}}^{0}$ and $\hat{\mathcal{I}}^{\perp}$ of the objects we have defined above. Our point is that the trees $S_{0}, S_{1}$ and $S$ remain to have the following properties with respect to the new versions of these objects,

$$
\hat{\mathcal{I}}^{0}=\operatorname{proj}\left[S_{0}\right] \text { and } \hat{\mathcal{I}}^{\perp}=\operatorname{proj}\left[S_{1}\right] .
$$

In particular, the ordinal $\alpha_{0}$ and the sequence of derivatives $\partial_{\alpha} S$ remains the same as well as the family $\mathcal{C}$ which we intend to manipulate using $(\mathrm{P})$.

By Lemma 8 and the fact that under (P) every subset of $\mathbb{N}^{\mathbb{N}}$ of size $\aleph_{1}$ is $\sigma$ bounded (see $[\mathrm{F}]$ ), there is $c_{0} \in \mathcal{I}$ such that

$$
c_{0} \backslash(\bigcup \mathcal{F}) \text { is infinite for every finite } \mathcal{F} \subseteq \mathcal{C} \cap \mathcal{I} .
$$


Using (P) and going to an infinite subset of $c_{0}$, we may assume that we actually have the following stronger property:

$$
c_{0} \cap c \text { is finite for every } c \in \mathcal{C} \cap \mathcal{I} \text {. }
$$

Pick a nonprincipal ultrafilter $\mathcal{U}$ on $\mathbb{N}$ such that $c_{0} \in \mathcal{U}$. Using $(\mathrm{P})$ we find an infinite set $a_{0} \subseteq c_{0}$ such that

$$
a_{0} \backslash c \text { is finite for all } c \in \mathcal{U} \cap(\mathcal{C} \cup-\mathcal{C}),
$$

where $-\mathcal{C}=\{\mathbb{N} \backslash c: c \in \mathcal{C}\}$. By sequential compactness of $K$, going to an infinite subset of $a_{0}$, we may assume that $a_{0}$ is actually a member of $\mathcal{I}^{0}$. Recall that we are assuming that $K$ is a compact subset of the first Baire class over $\mathbb{N}^{\mathbb{N}}$, so for a given $\varepsilon>0$ and finite $F \subseteq \mathbb{N}^{\mathbb{N}}$ the set

$$
b(F, \varepsilon)=\left\{n:\left|f_{n}(x)\right|<\varepsilon \text { for all } x \in F\right\}
$$

is simply the trace of a neighborhood of $\overrightarrow{0}$ with the countable dense set $\left\{f_{n}\right\}$. So the family of sets of the form $b(F, \varepsilon)$ is a natural basis of the dual-filter of $\mathcal{I}$. Note also that by $(\mathrm{G} 8)$, no member of the family $\mathcal{U} \cap \mathcal{C}$ belongs to $\mathcal{I}$, so the family

$$
\mathcal{F}(F, \varepsilon)=\{c \cap b(F, \varepsilon): c \in \mathcal{U} \cap \mathcal{C}\}
$$

has the finite intersection property. Using $(\mathrm{P})$ again we find infinite $a(F, \varepsilon)$ such that

$$
a(F, \varepsilon) \backslash b \text { is finite for all } b \in \mathcal{F}(F, \varepsilon) \text {. }
$$

Using sequential compactness of $K$ again, going to an infinite subset of $a(F, \varepsilon)$, we may assume that the sequence $\left\{f_{n}\right\}_{n \in a(F, \varepsilon)}$ converges to some point $z(F, \varepsilon)$ of $K$. It is clear that the subset

$$
\left\{z(F, \varepsilon): F \text { a finite subset of } \mathbb{N}^{\mathbb{N}}, \varepsilon>0\right\}
$$

of $K$ accumulates to $\overrightarrow{0}$, so using again the countable tightness of $K$ ([R3, Lemma 3.9]), we find a countable subset $\left\{z\left(F_{i}, \varepsilon_{i}\right): i \in \mathbb{N}\right\}$ of this set that still accumulates to $\overrightarrow{0}$. By (G10) the corresponding countable family

$$
\left\{a\left(F_{i}, \varepsilon_{i}\right): i \in \mathbb{N}\right\}
$$

of infinite sets of integers and the family $\mathcal{U} \cap \mathcal{C}$ form a pre-gap. Using $(\mathrm{P})$ we can find a set $d_{1}$ which interpolates this pre-gap, or in other words:

$$
\begin{aligned}
& a\left(F_{i}, \varepsilon_{i}\right) \backslash d_{1} \text { is finite for all } i, \\
& d_{1} \backslash c \text { is finite for all } c \in \mathcal{U} \cap \mathcal{C} .
\end{aligned}
$$

By (G11) the set of accumulation points of $\left\{f_{n}\right\}_{n \in d_{1}}$ includes each of the points $z\left(F_{i}, \varepsilon_{i}\right)(i \in \mathbb{N})$. So, in particular, $\overrightarrow{0}$ is an accumulation point of $\left\{f_{n}\right\}_{n \in d_{1}}$ and therefore by the Bourgain-Fremlin-Talagrand Theorem there is infinite $a_{1} \subseteq d_{1}$ such that $\left\{f_{n}\right\}_{n \in a_{1}}$ converges to $\overrightarrow{0}$. It follows that (see (G12)):

$$
\begin{aligned}
& a_{1} \in \mathcal{I}^{\perp}, \\
& a_{1} \backslash c \text { is finite for all } c \in \mathcal{U} \cap \mathcal{C} .
\end{aligned}
$$

Since $a_{0} \in \mathcal{I}$, the intersection $a_{0} \cap a_{1}$ is finite so removing it from one of the sets we may assume that $a_{0}$ and $a_{1}$ are actually disjoint. Since $\mathcal{I}^{0}=\operatorname{proj}\left[S_{0}\right]$ and $\mathcal{I}^{\perp}=\operatorname{proj}\left[S_{1}\right]$ we can find $b_{0}$ and $b_{1}$ such that $\left(a_{0}, b_{0}\right) \in\left[S_{0}\right]$ and $\left(a_{1}, b_{1}\right) \in\left[S_{1}\right]$. It 
follows that $\left(\left(a_{0}, b_{0}\right),\left(a_{1}, b_{1}\right)\right)$ is an infinite branch of $S$. So, the proof of Lemma 9 is finished once we show the following:

Claim 1. $\left(\left(a_{0}, b_{0}\right),\left(a_{1}, b_{1}\right)\right) \in\left[\partial_{\alpha_{0}} S\right]$.

Proof. Otherwise, there is $\alpha<\alpha_{0}$ such that

$$
\left(\left(a_{0}, b_{0}\right),\left(a_{1}, b_{1}\right)\right) \in\left[\partial_{\alpha} S\right] \backslash\left[\partial_{\alpha+1} S\right] .
$$

Pick a node $p=\left(\left(s_{0}, t_{0}\right),\left(s_{1}, t_{1}\right)\right)$ on this branch which does not belong to the subtree $\partial_{\alpha+1} S$. It follows that $\left(\left(a_{0}, b_{0}\right),\left(a_{1}, b_{1}\right)\right)$ belongs to $\left[\left(\partial_{\alpha} S\right)_{p}\right]$ and therefore

$$
a_{0} \subseteq c(\alpha, p) .
$$

Since $a_{0}$ is an infinite subset of $c_{0}$, from (G9) we conclude that the member $c(\alpha, p)$ of the family $\mathcal{C}$ rather than its complement must belong to $\mathcal{U}$. So, in particular, we have (see (G14)):

$$
a_{1} \backslash c(\alpha, p) \text { is finite. }
$$

By the definition of $c(\alpha, p)$ we can find an integer $k$ above the height of our node $p=\left(\left(s_{0}, t_{0}\right),\left(s_{1}, t_{1}\right)\right)$ and an infinite branch $\left(\left(e_{0}, h_{0}\right),\left(e_{1}, h_{1}\right)\right)$ of $\left(\partial_{\alpha} S\right)_{p}$ such that

$$
k \in a_{1} \cap e_{0} .
$$

Pick a large enough integer $m$ such that $k$ belongs to the intersection of the sets $a_{1}[m]$ and $e_{0}[m]$ formed by taking the initial part consisting of the first $m$ many members of the sets $a_{1}$ and $e_{0}$, respectively. Then

$$
\left(\left(a_{0}[m], b_{0}[m]\right),\left(a_{1}[m], b_{1}[m]\right)\right) \quad \text { and } \quad\left(\left(e_{0}[m], h_{0}[m]\right),\left(e_{1}[m], h_{1}[m]\right)\right)
$$

are the nodes of height $m$ belonging to the branches

$$
\left(\left(a_{0}, b_{0}\right),\left(a_{1}, b_{1}\right)\right) \quad \text { and } \quad\left(\left(e_{0}, h_{0}\right),\left(e_{1}, h_{1}\right)\right),
$$

respectively (and therefore belonging to $\partial_{\alpha} S$ ), that extend our initial node $p=$ $\left(\left(s_{0}, t_{0}\right),\left(s_{1}, t_{1}\right)\right)$ and witness the fact that $p \in \partial\left(\partial_{\alpha} S\right)=\partial_{\alpha+1} S$, a contradiction. This finishes the proof.

The following consequence of Lemma 9 gives us an object (a "Luzin gap") inside $\mathcal{I}^{0} \times \mathcal{I}^{\perp}$ which is all that is needed from now on to finish the proof of Theorem 9 .

Lemma 10. There is a sequence $\left(a_{x}, b_{x}\right)\left(x \in 2^{\mathbb{N}}\right)$ of elements of $\mathcal{I}^{0} \times \mathcal{I}^{\perp}$ such that

(i) $x \mapsto\left(a_{x}, b_{x}\right)$ is $1-1$ and continuous,

(ii) $a_{x} \cap b_{x}=\emptyset$,

(iii) $\left(a_{x} \cap b_{y}\right) \cup\left(b_{x} \cap a_{y}\right) \neq \emptyset$ whenever $x \neq y$.

Proof. Starting with $p^{\emptyset}=((\emptyset, \emptyset),(\emptyset, \emptyset)) \in \partial_{\alpha_{0}} S$ we build recursively an embedding

$$
\sigma \mapsto p^{\sigma}=\left(\left(s_{0}^{\sigma}, t_{0}^{\sigma}\right),\left(s_{1}^{\sigma}, t_{1}^{\sigma}\right)\right)
$$

such that for every $\sigma$

$$
p^{\sigma^{\frown} 0} \text { and } p^{\sigma^{\frown 1}} \text { are two extensions of } p^{\sigma} \text { belonging to the same level }
$$

$$
\text { of } \partial_{\alpha_{0}} S \text { and having the property that }
$$

$$
\left(s_{0}^{\sigma^{\frown} 0} \cap s_{1}^{\sigma^{\frown}}\right) \cup\left(s_{0}^{\sigma^{\frown} 1} \cap s_{1}^{\sigma^{\frown} 0}\right) \neq \emptyset .
$$


This is possible to do using the fact that $\partial\left(\partial_{\alpha_{0}} S\right)=\partial_{\alpha_{0}} S$. When this is done, for $x \in 2^{\mathbb{N}}$ we let

$$
a_{x}=\bigcup_{n=0}^{\infty} s_{0}^{x \uparrow n} \quad \text { and } \quad b_{x}=\bigcup_{n=0}^{\infty} s_{1}^{x \uparrow n} .
$$

It is clear that the so-defined map $x \mapsto\left(a_{x}, b_{x}\right)$ satisfies (i), (ii) and (iii).

Lemma 11. $\bigcup_{x \in P} a_{x} \notin \mathcal{I}$ for every uncountable $P \subseteq 2^{\mathbb{N}}$.

Proof. Suppose that for some uncountable $P \subseteq 2^{\mathbb{N}}$,

$$
a=\bigcup_{x \in P} a_{x}
$$

belongs to $\mathcal{I}$. Then we can find uncountable $P_{0} \subseteq P, n \in \mathbb{N}$ and $s, t \subseteq\{0, \ldots, n\}$ such that

$$
\begin{array}{ll}
a \cap b_{x} \subseteq\{0, \ldots, n\} & \text { for all } x \in P_{0}, \\
a_{x} \cap\{0, \ldots, n\}=s \text { and } b_{x} \cap\{0, \ldots, n\}=t & \text { for all } x \in P_{0} .
\end{array}
$$

Then for $x$ and $y$ in $P_{0}$ we have

$$
\left(a_{x} \cap b_{y}\right) \cup\left(b_{x} \cap a_{y}\right)=\emptyset,
$$

contradicting (iii) of Lemma 10. This finishes the proof.

The Cantor space $2^{\mathbb{N}}$ has a natural lexicographical ordering and a metric defined via the mapping

$$
\Delta(x, y)=\min \{n: x(n) \neq y(n)\} .
$$

A lexicographically increasing sequence $\left\{x_{n}\right\}$ of elements of $K$ is convex if it has the following property:

$$
\Delta\left(x_{n}, x_{n+1}\right)<\Delta\left(x_{n+1}, x_{n+2}\right) \quad \text { for all } n .
$$

This notion can of course be defined in any metric space $M$ which has a total ordering that is sufficiently compatible with the metric such as for example $\mathbb{R}, \mathbb{N}^{\mathbb{N}}$, etc. Some care, however, is needed when extending this definition into general context since the condition (G22) relies on the fact that $d(x, y)=2^{-\Delta(x, y)}$ is an ultrametric, a metric with a rather special property. For example, in the case $M=\mathbb{R}$ we say that a strictly increasing sequence $\left\{x_{n}\right\}$ is convex if its supremum $x_{\infty}$ is $<\infty$ and if

$$
x_{n+1}-x_{n}>x_{\infty}-x_{n+1} \quad \text { for all } n .
$$

Whenever we have an ordered metric space $M$ for which this notion can be defined, we let $M^{[\infty]}$ denote the space of all infinite increasing convex sequences of $M$ with the topology induced from the infinite power of $M$. The reason for not considering the space of all sequences of $M$ or all strictly increasing sequences of $M$ is that we would like to have coloring theorems for these infinite powers and any failure of convexity conditions such as (G22) or (G23) gives us a way to define even a continuous colorings without monochromatic infinite powers of nonscattered sets. We shall use the following coloring result of Louveau, Shelah and Velickovic [LSV] which has a close relationship with the Ramsey-theoretic results exposed above in $\S \mathrm{C}$. 
Lemma 12. For every analytic subset $\mathcal{A}$ of $\left(2^{\mathbb{N}}\right)^{[\infty]}$ there is a perfect set $P \subseteq 2^{\mathbb{N}}$ such that $P^{[\infty]}$ is included in $\mathcal{A}$ or its complement.

Of course, by symmetry we have the similar coloring theorem for the space $\left(2^{\mathbb{N}}\right)^{[-\infty]}$ of all infinite decreasing convex sequences of the Cantor cube.

Recall that for every $x \in 2^{\mathbb{N}}$ the set $a_{x}$ belongs to $\mathcal{I}^{0}$, so the limit

$$
g_{x}=\lim _{n \in a_{x}} f_{n}
$$

exists and is $\neq \overrightarrow{0}$. We shall select the set $D$ such that $D \cup\{\overrightarrow{0}\}$ satisfies the conclusion of Theorem 8 among the elements of the family $g_{x}\left(x \in 2^{\mathbb{N}}\right)$. It is therefore quite natural to consider the following two collections:

$$
\begin{aligned}
& \mathfrak{X}^{+}=\left\{\left\{x_{n}\right\} \in\left(2^{\mathbb{N}}\right)^{[\infty]}:\left\{g_{x_{n}}\right\} \text { accumulates to } \overrightarrow{0}\right\}, \\
& \mathfrak{X}^{-}=\left\{\left\{x_{n}\right\} \in\left(2^{\mathbb{N}}\right)^{[-\infty]}:\left\{g_{x_{n}}\right\} \text { accumulates to } \overrightarrow{0}\right\} .
\end{aligned}
$$

The following lemma is a place where Lemma 6 (as well as Lemmas 11 and 12) is crucially used.

Lemma 13. There is a perfect subset $P$ of $2^{\mathbb{N}}$ such that $P^{[\infty]}$ is included in $\mathfrak{X}^{+}$ while $P^{[-\infty]}$ is included in $\mathfrak{X}^{-}$.

Proof. By Lemma 12 and by symmetry it suffices to show that

$$
P^{[\infty]} \cap \mathfrak{X}^{+} \neq \emptyset \text { for every perfect set } P \subseteq 2^{\mathbb{N}} .
$$

To establish this we shall need among other things the following reformulation of Lemma 11:

$$
\left\{g_{x}: x \in R\right\} \text { accumulates to } \overrightarrow{0} \text { for every uncountable set } R \subseteq P \text {. }
$$

To see that this is indeed true, suppose that there is a neighborhood $B(F, \varepsilon)$ of $\overrightarrow{0}$ whose closure misses $\left\{g_{x}: x \in R\right\}$ for some uncountable set $R \subseteq P$. Then

$$
a=\left\{n \in \mathbb{N}: f_{n} \notin B(F, \varepsilon)\right\}
$$

is a member of $\mathcal{I}$ with the property that

$$
a_{x} \backslash a \text { is finite for all } x \in R \text {. }
$$

Then there is uncountable $R_{0} \subseteq R$ and $n \in \mathbb{N}$ such that

$$
a_{x} \subseteq a \cup\{0, \ldots, n\} \quad \text { for all } x \in R_{0} .
$$

This contradicts the conclusion of Lemma 11. Let

$$
Q=P \backslash\{\max (P)\},
$$

and for $x \in Q$, let

$$
Q_{x}=\{y \in Q: y>x\} \quad \text { and } \quad D_{x}=\left\{g_{y}: y \in Q_{x}\right\},
$$

where $>$ and max refer to the lexicographical ordering of $2^{\mathbb{N}}$. Since each of the sets $Q_{x}$ is uncountable, by (G25) we know that

$$
D_{x} \text { accumulates to } \overrightarrow{0} \text { for all } x \in Q \text {. }
$$

Since this is a monotone family of subsets of $K$, there is an ultrafilter $\mathcal{U}$ on $K$ which extends the neighborhood filter of $\overrightarrow{0}$ and contains $D_{x}$ for all $x \in Q$. By Lemma 6 there is a decreasing sequence $\left\{B_{n}\right\}$ of elements of $\mathcal{U}$ which converges to 
$\overrightarrow{0}$, i.e., every neighborhood of $\overrightarrow{0}$ contains all but finitely many of the $B_{n}$ 's. Let $x_{-1}=\min (Q)$. The set $D_{x_{-1}} \cap B_{0}$ is nonempty so we can pick an element of the form $g_{x_{0}}$ in this set. Now, the set $D_{x_{0}} \cap B_{1}$ is nonempty so we can pick an element of the form $g_{x_{1}}$ in this set, and so on. Continuing this process will give us a strictly increasing infinite sequence $\left\{x_{n}\right\}$ of elements of $Q$ such that

$$
g_{x_{n}} \in B_{n} \quad \text { for all } n \text {. }
$$

Going to an infinite sequence we may assume that $\left\{x_{n}\right\}$ is actually a convex increasing sequence, i.e., a member of $P^{[\infty]}$. By the choice of the sequence $\left\{B_{n}\right\} \subseteq \mathcal{U}$ from (G29) we conclude that the sequence $\left\{g_{x_{n}}\right\}$ converges to $\overrightarrow{0}$. It follows that

$$
\left\{x_{n}\right\} \in P^{[\infty]} \cap \mathfrak{X}^{+},
$$

so the intersection is nonempty.

Lemma 14. There is a perfect subset $P$ of $2^{\mathbb{N}}$ such that $\left\{g_{x}: x \in R\right\}$ accumulates to $\overrightarrow{0}$ for every infinite subset $R$ of $P$.

Proof. We shall show that any perfect subset $P$ of $2^{\mathbb{N}}$ that satisfies Lemma 13 also satisfies the conclusion of Lemma 14. Otherwise there is an open neighborhood $B(F, \varepsilon)$ of $\overrightarrow{0}$ such that

$$
R=\left\{x \in P: g_{x} \notin B(F, \varepsilon)\right\}
$$

is infinite. Every such infinite set $R \subseteq P$ contains either a convex increasing or a convex decreasing sequence $\left\{x_{n}\right\}$. Such a sequence $\left\{x_{n}\right\}$ will be a member of either $P^{[\infty]}$ or $P^{[-\infty]}$ and therefore a member of either $\mathfrak{X}^{+}$or $\mathfrak{X}^{-}$. In any case, from the definition of these two sets, we conclude that the corresponding sequence $\left\{g_{x_{n}}\right\}$ of points of $K$ must accumulate to $\overrightarrow{0}$, a contradiction. This finishes the proof.

Let $P$ be the perfect subset of $2^{\mathbb{N}}$ satisfying the conclusion of Lemma 14 and let

$$
D=\left\{g_{x}: x \in P\right\} .
$$

By Lemma 14, the mapping $x \mapsto g_{x}$ is finite-to-one on $P$, so the set $D$ has size continuum. By Lemma 14 again every neighborhood of $\overrightarrow{0}$ contains all but finitely many elements of $D$. Hence $D \cup\{\overrightarrow{0}\}$ is a one-point compactification of the discrete space $D$ of size continuum with $\overrightarrow{0}$ as its point at infinity. This finishes the proof of Theorem 9 .

\section{REFERENCES}

[AU] P. Alexandroff, P. Urysohn, Mèmoire sur les espaces topologiques compacts, Verh. Akad. Wetensch. Amsterdam 14 (1929).

$[\mathrm{AM}]$ S. Argyros and S. Mercourakis, On weakly Lindelöf Banach spaces, Rocky Mountain J. Math. 23 (1993), 395-446. MR 94i:46016

[Ba] R. Baire, Sur les fonctions des variables rèelles, Ann. Mat. Pura Appl. 3 (1899), 16-30.

[Bl] A. Blass, A partition theorem for perfect sets, Proc. Amer. Math. Soc. 82 (1981), 271-277. MR 83k:03063

[Bo] J. Bourgain, Some remarks on compact sets of first Baire class, Bull. Soc. Math. Belg. 30 (1978), 3-10. MR 80j:54008

[BFT] J. Bourgain, D.H. Fremlin and M. Talagrand, Pointwise compact sets of Baire-measurable functions, Amer. J. Math. 100 (1978), 845-886. MR 80b:54017

[CP] E. Čech, B. Pospišil, Sur les espaces compacts, Publ. Fac. Sci. Univ. Masaryk Brno 258 (1938), 3-7. 
[D] G. Debs, Effective properties in compact sets of Borel function, Mathematika 34 (1987), 64-68. MR 89b:03082

[F1] D. H. Fremlin, Consequences of Martin's axiom, Cambridge University Press, 1984. MR 86i:03001

[F] D.H. Fremlin, On compact sets carrying Radon measure of uncountable Maharam type, Fund. Math. 154 (1997), no. 3, 295-304. MR 99d:28019

[Go] G. Godefroy, Compacts de Rosenthal, Pacific J. Math. 91 (1980), 293-306. MR 82f:54030

[GL] G. Godefroy and A. Louveau, Axioms of determinacy and biorthogonal systems, Israel J. Math. 67 (1989), 109-116. MR 91k:03126

[GT] G. Godefroy and M. Talagrand, Espaces de Banach representables, Israel J. Math. 41 (1982), 321-330. MR 84g:46019

[Gr] G. Gruenhage, Perfectly normal compacta, cosmic spaces, and some partition problems, in: Open Problems in Topology (J. van Mill and G.M. Reed, eds.), Elsevier Sci. Publ., Amsterdam, 1990. MR 92c:54001

[H] F. Hausdorff, Die Graduierung nach dem Endrerlauf, Abl. Königl. Sächs Gesell. Wiss Math.-Phys. Kl. 31 (1909), 296-334.

[J] R.C. James, A separable somewhat reflexive Banach space with non-separable dual, Bull. Amer. Math. Soc. 80 (1974), 738-743. MR 54:5811

[Ke] A.S. Kechris, Classical descriptive set theory, Springer-Verlag, New York 1995. MR 96e:03057

[Kr] A. Krawczyk, Rosenthal compacta and analytic sets, Proc. Amer. Math. Soc. 115 (1992), 1095-1100. MR 92j:54050

[LS] J. Lindenstrauss and C. Stegall, Examples of separable spaces which do not contain $\ell^{1}$ and whose duals are non-separable, Studia Math. 54 (1975), 81-105. MR 52:11543

[LT] J. Lindenstrauss and L. Tzafriri, Classical Banach Spaces I, Springer-Verlag, 1977. MR 58:17766

[LSV] A. Louveau, S. Shelah and B. Velickovic, Borel partitions of infinite subtrees of a prefect tree, Ann. Pure Appl. Logic 63 (1993), 271-281. MR 94g:04003

[Lo] S. Lojasiewicz, An introduction to the theory of real functions, Wiley-Interscience Publ., New York 1988. MR 89e:26001

[M1] W. Marciszewski, On a classification of pointwise compact sets of the first Baire class functions, Fund. Math. 133 (1989), 195-209. MR 91k:54026

[M2] W. Marciszewski, On properties of Rosenthal compacta, Proc. Amer. Math. Soc. 115 (1992), 797-805. MR 92i:54023

$[\mathrm{MN}]$ S. Mercourakis and S. Negrepontis, Banach spaces and Topology II, in: Recent Progress in Topology (M. Hušek and J. van Mill, eds.), Elsevier Sci. Publ., New York, 1992. MR 95g:54004

[Mi] A.W. Miller, Infinite combinatorics and definability, Ann. Pure Appl. Logic 41 (1989), 179-203. MR 90b:03070

[N] I.P. Nathanson, Theory of functions of real variable, Moscow, 1950.

[Na] I. Namioka, Separate continuity and joint continuity, Pacific J. Math. 51 (1974), 515-531. MR 51:6693

[OR] E. Odell and H.P. Rosenthal, A double-dual characterization of separable Banach spaces containing $\ell^{1}$, Israel J. Math. 20 (1975), 375-384. MR 51:13654

[Pa] J. Pawlikowski, Parametrized Ellentuck Theorem, Topology and its Appl. 37 (1990), 65-73. MR 91j:04002

[Po1] R. Pol, Note on the spaces $P(S)$ of regular probability measures whose topology is determined by countable subsets, Pacific J. Math. 100 (1982), 185-201. MR 83g:54024

[Po2] R. Pol, On pointwise and weak topology in function spaces, Warszaw University, 1984.

[Po3] R. Pol, Note on Pointwise convergence of sequences of Analytic sets, Mathematika 36 (1989), 290-300. MR 91j:54070

[R1] H.P. Rosenthal, A characterization of Banach spaces containing $\ell^{1}$, Proc. Nat. Acad. Sci. USA 71 (1974), 2411-2413. MR 50:10773

[R2] H.P. Rosenthal, Pointwise compact subsets of the first Baire class, Amer. J. Math. 99 (1977), 362-378. MR 55:11032

[R3] H.P. Rosenthal, Some recent discoveries in the isomorphic theory of Banach spaces, Bull. Amer. Math. Soc. 84 (1978), 803-831. MR 80d:46023 
[Ro] F. Rothberger, Sur la familles indenombrables de suites de nombres naturels et les problemes concernant la propriete C, Proc. Cambridge Phil. Soc. 37 (1941), 109-126. MR 2:352a

[S] D. Scott, A proof of the independence of the Continuum Hypothesis, Math. Systems Theory 1 (1966), 89-111. MR 36:1321

[St] C. Stegall, The Radon-Nikodym property in conjugate Banach spaces, Trans. Amer. Math. Soc. 206 (1975), 213-223. MR 51:10581

[Ste] J. Stern, A Ramsey theorem for trees with an application to Banach spaces, Israel J. Math. 29 (1978), 179-188. MR 57:16114

[Sz] W. Szlenk, The non-existence of a separable reflexive Banach space universal for all separable reflexive Banach spaces, Studia. Math. 30 (1968), 53-61. MR 37:3327

[Ta] M. Talagrand, Pettis integral and measure theory, Memoirs of the AMS, Vol 51, No. 307, 1984. MR 86j: 46042

[To1] S. Todorcevic, Partition problems in topology, Amer. Math. Soc., Providence, 1989. MR 90d:04001

[To2] S. Todorcevic, Free sequences, Topology and its Appl. 35 (1990), 235-238. MR 91f:54003

[To3] S. Todorcevic, Analytic gaps, Fund. Math. 150 (1996), 55-66. MR 98j:03070

[W] H.E. White, Jr., Variants of Blumberg's Theorem, Illinois J. Math. 26 (1982), 359-373. MR 83g:54034

Department of Mathematics, University of Toronto, Toronto, Ontario, Canada M5S $3 \mathrm{G} 3$

E-mail address: stevo@math.toronto.edu 